\title{
Multimedia Fate and Human Intake Modeling: Spatial versus Non- Spatial Insights for Chemical Emissions in Western Europe
}

David W. Pennington, Manuele Margni, Christoph Ammann, Olivier Jolliet

\section{Supporting Information}

Copies of the Western European version of these models as referred to in this paper and supporting reports are available freely upon request from the authors in Excel format (e.g. IMPACT 2002, www.epfl.ch/impact).

Background reports ${ }^{1}, \mathrm{PhD}$ dissertations ${ }^{2,3}$, and Diploma dissertations ${ }^{4,5}$ that provide further detail related to the model developments are also freely available from the authors.

\section{Contents of Supporting Information}

1. Property data and selection of representative chemicals (for Figure 6)

2. PeCDF Property Data, Spatial Emissions Data, and Intake Distribution Plots (for evaluation using monitoring data)

3. Property data for DDT, Toluene, Propachlor, and Dicofol (for Figure 7)

4. Model sensitivity analyses

5. Differences between Predictions and Monitoring Observations Discussion

6. River Flow Rates Comparison

7. Modeled Environmental Compartments

8. Use of watershed versus grid delimitations for the atmospheric compartments

9. List of Lakes and Lake Parameters

10. Default Uniform and Non-Spatial Version Model Parameters

11. Algebraic Chemical Fate Modelling Mathematical Solutions

12. Summary of Fate Rate Coefficient Algorithms

13. Exposure Rate Coefficients

14. Estimating bioaccumulation factors for organic compounds

15. Food production rate modifications for livestock feeds

16. References for supporting information

\section{Property data and selection of representative chemicals (for Figure 6)}

Chemicals in Table 1 were selected from approximately 500 non-dissociating organic chemicals using data adopted analogous to US EPA's draft WMPT tool data selection hierachy ${ }^{6}$. Data are from, in order of typically preference adopted, Mackay et al. data compilation handbooks ${ }^{7}$, Howard et al. data compilation handbooks ${ }^{8,9}$, Physprop experimental data ${ }^{10}$, Epiwin experimental data ${ }^{11}$, Physprop estimated data ${ }^{10}$, Epiwin estimated data ${ }^{11}$. Data gaps were additionally filled using the CalTox model ${ }^{12}$ and the USES-LCA model ${ }^{13}$ databases.

Data should be considered illustrative and were adopted to reflect the likely behaviour of organic chemicals in general, rather than to reflect the nature of specific substances. 
Chemicals were classified in terms of their likely partitioning in the environment and in terms of likely exposure pathways for human health. These were then overlaid to identify test subgroups. Chemicals were also ranked in terms of their likely overall tendency to be persistent and to be subject to long range atmospheric transport (LRT) in the environment. Four sub-lists were then identified, one for each of the 14 partitioning/pathway zones - accounting for LRT only, persistence only, both LRT \& persistence, neither LRT nor persistence tendencies. A total of 56 test zones were identified. For each, where possible, at least one organic chemical was selected for consideration.

Table 1: Input data for selected non-dissociating and non-amphiphilic organic chemicals for the subset covering all relevant combinations according to the selection criteria. $^{14}$

\begin{tabular}{|c|c|c|c|}
\hline Chemical & \begin{tabular}{|c|} 
Henry's \\
Constant \\
(Pa m3 \\
mol-1) \\
\end{tabular} & Log Kow & $\begin{array}{c}\text { Molecular } \\
\text { Mass } \\
\text { (g/mole) }\end{array}$ \\
\hline Tetrachloroethylene & 1742 & 2.576341 & 166 \\
\hline Carbon tetrachloride & 3248 & 2.6 & 154 \\
\hline 1,3-Butadiene & 257000 & 2 & 54.09 \\
\hline Methomyl & $1.9 \mathrm{E}-08$ & 0.6 & 162 \\
\hline Acephate & 5 E-11 & -1 & 183 \\
\hline Formaldehyde & 0.03 & 0.35 & 30 \\
\hline PCBS & 35 & 6.3 & 292 \\
\hline Di(n-octyl) phthalate & 0.1 & 8.1 & 391 \\
\hline Benzene, hexabromo- & 3 & 6.1 & 552 \\
\hline Cypermethrin & $2 \mathrm{E}-05$ & 6.6 & 416 \\
\hline Mirex & 0.1 & 5.3 & 545 \\
\hline Trifluralin & 3 & 5.3 & 336 \\
\hline Dicofol & $6 \mathrm{E}-05$ & 5 & 370 \\
\hline p-Dichlorobenzene & 297 & 3.5 & 147 \\
\hline Aldrin & 11 & 3 & 365 \\
\hline 1,1,2,2-Tetrachloroethane & 26 & 2.4 & 168 \\
\hline Captan & 0.7 & 2.3 & 301 \\
\hline Pronamide & 0.5 & 3.5 & 256 \\
\hline Anthracene & 4.3 & 4.5 & 178 \\
\hline gamma-Hexachlorocyclohexane & 0.3 & 3.7 & 291 \\
\hline Dimethyl phthalate & 0.2 & 1.7 & 194 \\
\hline Methanol & 0.5 & -0.77 & 32 \\
\hline 1,2-Dichloroethane & 117 & 1.4 & 99 \\
\hline Ethyl acetate & 14 & 0.7 & 88 \\
\hline N-Nitrosodiethylamine & 0.18 & 0.48 & 102 \\
\hline Thioperoxydicarbonic diamide, tetramethyl- & 0.008 & 1.7 & 240 \\
\hline Propoxur & $4.5 \mathrm{E}-05$ & 1.5 & 209 \\
\hline 1H-Isoindole-1,3(2H)-dione, 2- (trichloromethyl)thio & 0.0004 & 3.6 & 297 \\
\hline Benomyl & $1.9 \mathrm{E}-09$ & 2.3 & 290 \\
\hline Hexachlorobutadiene & 2407 & 4.7 & 261 \\
\hline Hexachlorocyclopentadiene & 2199 & 5.1 & 273 \\
\hline
\end{tabular}

Supporting Information, Pennington et al. "Spatial versus non-spatial modelling" 


\begin{tabular}{|l|r|r|r|}
\hline Heptachlor epoxide & 150 & 5.1 & 389 \\
\hline Hexachlorobenzene & 78 & 5.5 & 285 \\
\hline Heptachlor & 22 & 5.3 & 373 \\
\hline
\end{tabular}

\begin{tabular}{|c|c|c|c|c|}
\hline \multirow{2}{*}{ Chemical } & \multirow[b]{2}{*}{ troposphere } & \multirow[b]{2}{*}{$\begin{array}{c}\text { Water } \\
\text { column }\end{array}$} & \multirow[b]{2}{*}{ soil } & \multirow[b]{2}{*}{ Sediment } \\
\hline & & & & \\
\hline Tetrachloroethylene & 23 & \begin{tabular}{|r|}
550 \\
\end{tabular} & 1700 & 5500 \\
\hline Carbon tetrachloride & 708 & 17000 & 5500 & 17000 \\
\hline 1,3-Butadiene & 0.2 & 5 & 550 & 1700 \\
\hline Methomyl & 23 & 550 & 550 & 5500 \\
\hline Acephate & 0.3 & 7.550623 & 52.8 & 52.8 \\
\hline Formaldehyde & 0.2 & 5 & 55 & 170 \\
\hline PCBS & 16 & 385.0818 & 900 & 3600 \\
\hline Di(n-octyl) phthalate & 1.1 & \begin{tabular}{|l|}
26.88 \\
\end{tabular} & 336 & 6540 \\
\hline Benzene, hexabromo- & 934 & 22415 & 1440 & 5760 \\
\hline Cypermethrin & 0.4 & 10 & 1247 & 1247 \\
\hline Mirex & 7 & 170 & 55000 & 55000 \\
\hline Trifluralin & 7 & 170 & 1700 & 5500 \\
\hline Dicofol & 3 & 70 & 1459 & 384 \\
\hline p-Dichlorobenzene & 23 & 550 & 5500 & 17000 \\
\hline Aldrin & 0.21 & 5 & 17000 & 55000 \\
\hline 1,1,2,2-Tetrachloroethane & 708 & 17000 & 5500 & 17000 \\
\hline Captan & 0.71 & 17 & 550 & 550 \\
\hline Pronamide & 57 & 1375 & 1932 & 180 \\
\hline Anthracene & 2.3 & 55 & 5500 & 17000 \\
\hline gamma-Hexachlorocyclohexane & 7.1 & 170 & 17000 & 55000 \\
\hline Dimethyl phthalate & 7.1 & 170 & 550 & 1700 \\
\hline Methanol & 7.1 & 170 & 55 & 170 \\
\hline 1,2-Dichloroethane & 71 & 1700 & 5500 & 17000 \\
\hline Ethyl acetate & 2.3 & 55 & 170 & 550 \\
\hline N-Nitrosodiethylamine & 0.21 & 5 & 1700 & 5500 \\
\hline Thioperoxydicarbonic diamide, tetramethyl- & 7.1 & 170 & 550 & 1700 \\
\hline Propoxur & 0.21 & 5 & 550 & 1700 \\
\hline 1H-Isoindole-1,3(2H)-dione, 2- (trichloromethyl)thio - & 1.1 & 27 & 13761 & 13761 \\
\hline Benomyl & 0.21 & 5 & 1700 & 5500 \\
\hline Hexachlorobutadiene & 716 & 17190 & 1704 & 1704 \\
\hline Hexachlorocyclopentadiene & 0.2 & 5 & 420 & 1680 \\
\hline Heptachlor epoxide & 1.4 & 33 & 7020 & 96 \\
\hline Hexachlorobenzene & 708 & 17000 & 55000 & 55000 \\
\hline Heptachlor & 2.3 & 55 & 1700 & 5500 \\
\hline
\end{tabular}

Supporting Information, Pennington et al. "Spatial versus non-spatial modelling" 


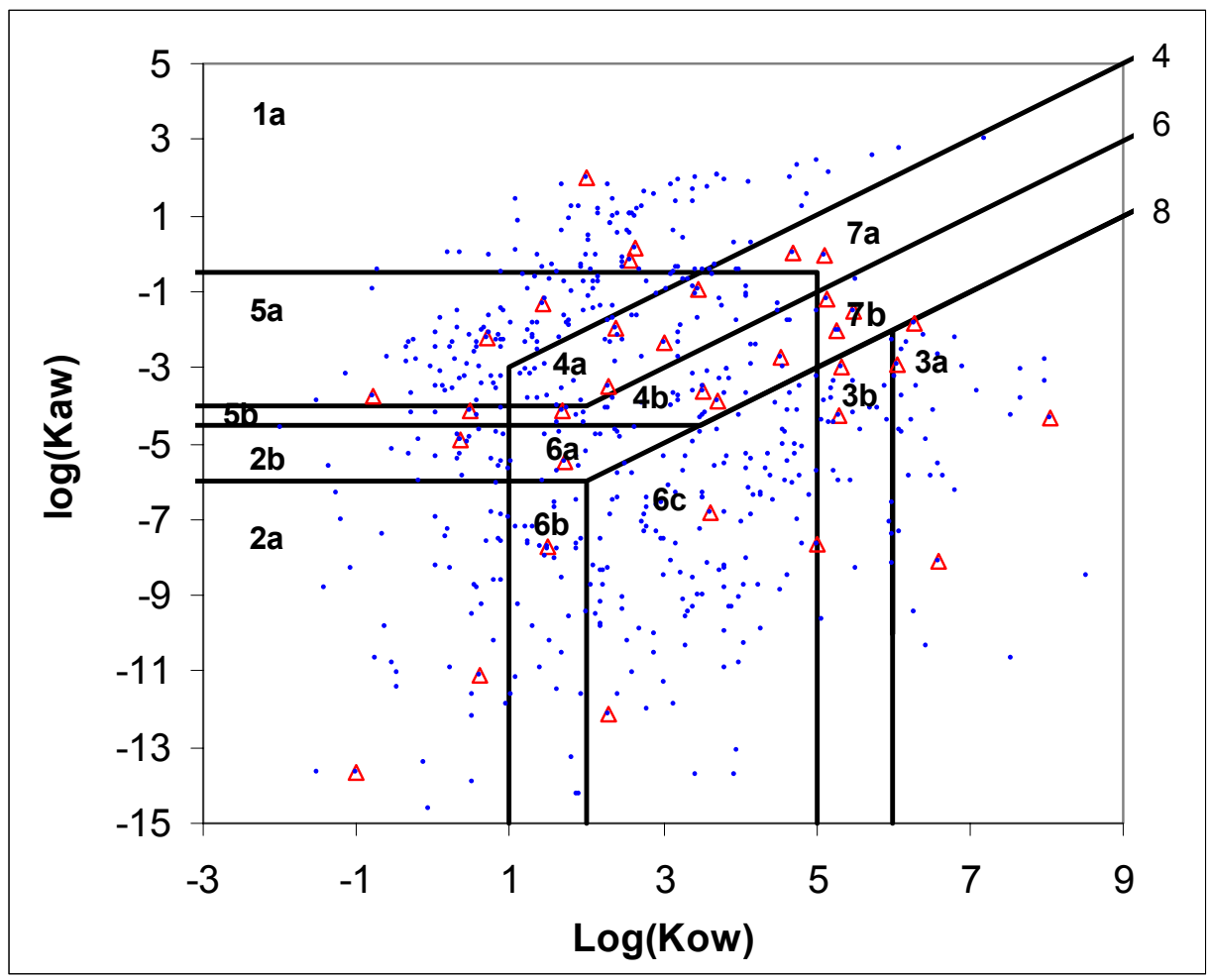

Figure 1: Octanol-water $\left(\mathrm{K}_{\mathrm{ow}}\right)$ vs. air-water $\left(\mathrm{K}_{\mathrm{aw}}\right)$ partitioning plot with the 14 partitioning/pathway zones. ${ }^{14}$ Blue points represent the $500+$ chemicals considered, red triangles indicate the selected representative set of organic, non-dissociating chemicals.

\section{Adopted property data, spatial emissions data and example intake distribution plots for PeCDF (for evaluation using monitoring data)}

Table 2: Adopted properties of PeCDF (based on Mackay et al. ${ }^{7}$ and Sinkkonen ${ }^{15}$, unless otherwise noted). Empirical data from McLachlan et al. ${ }^{16}$ were available and used to estimate biotransfer to milk instead of using correlations.

\begin{tabular}{l|l|l}
$\begin{array}{c}\text { Henry's Law constant } \\
\mathrm{Pa} \mathrm{m}^{3} \text { mole }^{-1}\end{array}$ & \multicolumn{2}{|c|}{ Octanol-water partitioning coefficient } \\
- & - & $\begin{array}{c}\text { BTF }(\text { milk })^{16} \\
\text { L day }^{-1}\end{array}$ \\
\hline 0.5 & $6 \times 10^{6}$ & 0.005
\end{tabular}

Degradation half-lives (days):

\begin{tabular}{c|c|c|c|c} 
In air & In water & In soil & In sediment & In vegetation $^{\Phi}$ \\
\hline 28 & 550 & $23000^{*}$ & $23000^{*}$ & $23000^{*}$ \\
$*$ denotes essentially negligible \\
estimated from half-life in soils.
\end{tabular}

Supporting Information, Pennington et al. "Spatial versus non-spatial modelling" 


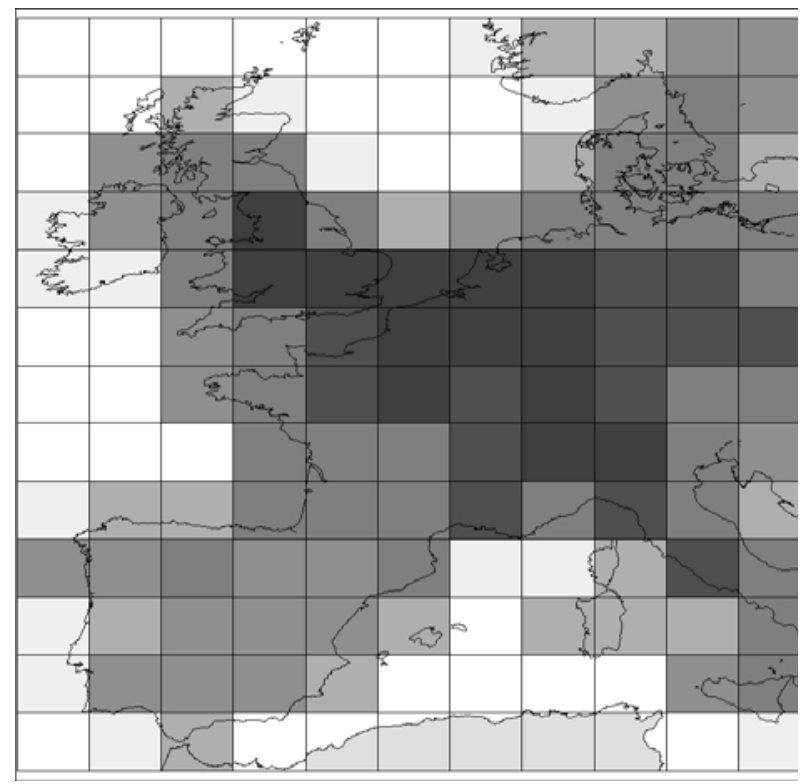

\section{Emission of PCDF [mg/hour]}

$\square 10$ to 25
5 to 10
2 to 5
1 to 2
0.5 to 1
0.2 to 0.5
0 to 0.2

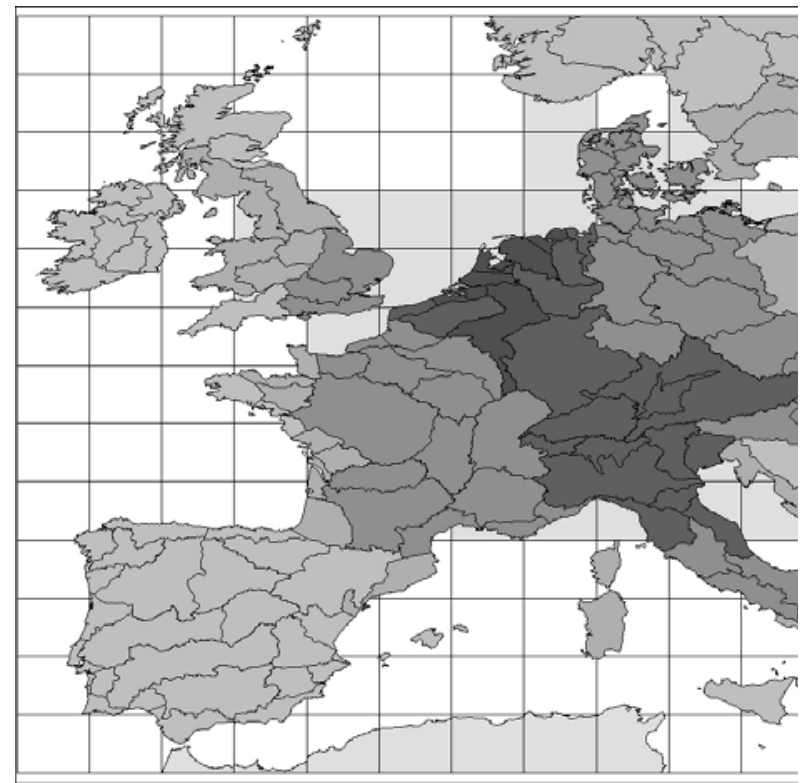

Intake per area of PCDF [kg10^-9/km2year]

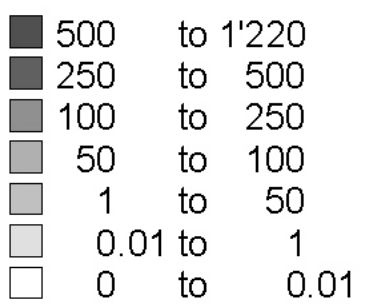

Figure 2: Spatial emission scenario of PeCDF based on data of Pacyna et al. ${ }^{17}$, as transformed to provide estimates of the emissions in each of the modeled air compartments (top map), and model results (bottom map) for the intake contributions per unit area within Western Europe attributable to each of the different watersheds and oceanic grid zones.

3.Key property data adopted for DDT, Toluene, Propachlor, and Dicofol (used in Figure 7)

Table 3: Properties of DTT, Toluene, Propachlor, and Dicofol (Mackay et al. ${ }^{18}$, USES-LCA ${ }^{13}$, CalTox $^{12}$ )

Supporting Information, Pennington et al. "Spatial versus non-spatial modelling" 


\begin{tabular}{|l|l|l|l|l|}
\hline & DTT & Toluene & Propachlor & Dicofol \\
\hline Molecular Mass [g/mole] & 355 & 92 & 212 & 370 \\
\hline $\begin{array}{l}\text { Henry's Constant [Pa m3 } \\
\text { mol-1] }\end{array}$ & 11 & 917 & $1 \mathrm{E}-05$ & $5.7 \mathrm{E}-05$ \\
\hline Log Kow & 6.2 & 2.7 & 2.2 & 5 \\
\hline $\begin{array}{l}\text { atmospheric degradation } \\
\text { half life [hours] }\end{array}$ & 170 & 17 & 17 & 70 \\
\hline $\begin{array}{l}\text { degradation half life in } \\
\text { water [hours] }\end{array}$ & 5500 & 550 & 186 & 899 \\
\hline $\begin{array}{l}\text { degradation half life in } \\
\text { soils [hours] }\end{array}$ & 17000 & 78 & 147 & 1460 \\
\hline $\begin{array}{l}\text { degradation half life in } \\
\text { sediment [hours] }\end{array}$ & 55000 & 230 & 147 & 384 \\
\hline
\end{tabular}

\section{Model sensitivity analyses}

For emissions to the air and the water, the organic chemicals were analyzed as a function of two key physical-chemical properties (see Figures 3 and 4). The strongest indicators of the partitioning of chemicals amongst different media is the Henry's Law constant, $\mathrm{H}$ - the air water partitioning coefficient - and the octanol-water partitioning coefficient, $\mathrm{K}_{\mathrm{ow}}$. For the two emission scenarios the chemicals were therefore plotted on a $\mathrm{H}$ vs. $\mathrm{K}_{\mathrm{ow}}$ graph, noting that diagonal octanol-air - Koa - lines could be added. Analogous results were presented by Gouin et al. ${ }^{19}$ for chemical fate and Bennett et al. ${ }^{20}$ for human exposure.

Emissions to soils were not addressed, as spatial variation was not represented for compartments in this medium.

Air emissions:

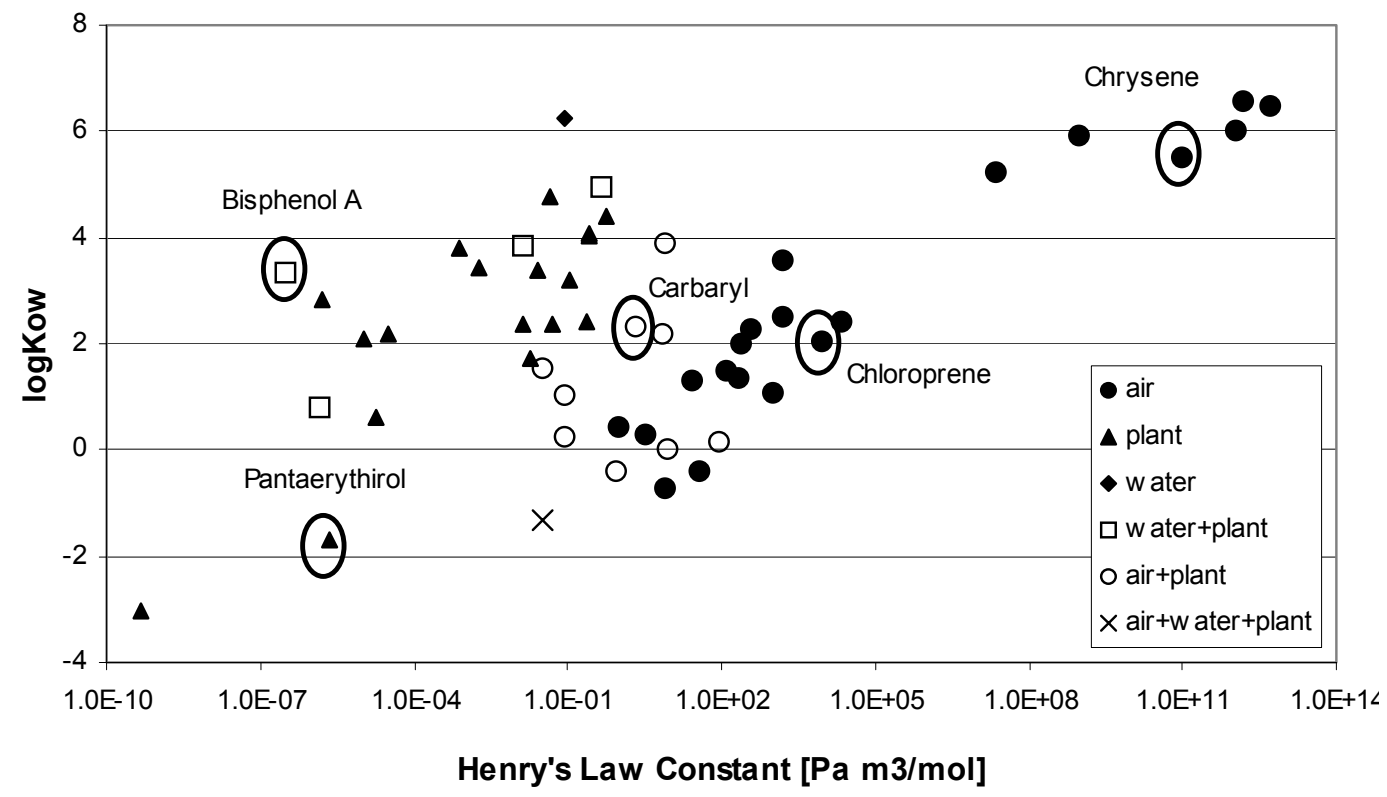

Figure 3: Uniform air emission for 53 organic chemicals plotted as a function of octanol-water partitioning coefficient (Kow) and Henry's Law constant (see Tables 4

Supporting Information, Pennington et al. "Spatial versus non-spatial modelling" 
and 5 at end of this section). The symbols denote the key environmental media that contributed to the intake in this model.

In general, chemicals with a high Henry's Law constant stayed in the atmosphere and the intake was dominated by inhalation. Chloroprene for example, a volatile chemical with $\mathrm{H}=21500[\mathrm{~Pa} \mathrm{~m} / \mathrm{mol}]$ remained predominantly in the atmosphere. The intake was predominantly associated with the concentrations in air. All the products with an $\mathrm{H}>100[\mathrm{~Pa} \mathrm{~m} / \mathrm{mol}]$ were dominated by inhalation in this model.

Chemicals with smaller $\mathrm{H}$ started to partition to plants and to the water. Chemicals like Carbaryl with $\mathrm{H}$ between 1 and $100\left[\mathrm{~Pa} \mathrm{~m}^{3} / \mathrm{mol}\right]$ were strongly influenced by the concentrations in both air and plants. Most of the chemicals with $\mathrm{H}<1\left[\mathrm{~Pa} \mathrm{~m}^{3} / \mathrm{mol}\right]$ were dominated by the vegetation. The intake by the atmosphere wasn't important anymore.

The intake of compounds like Pantaerythirol with a small $\mathrm{H}$ and a small $\mathrm{K}_{\mathrm{ow}}$ were dominated by the concentrations in vegetation. If $\mathrm{K}_{\mathrm{ow}}$ and the half-life time in the water are high, like for Bisphenol A for example, the water compartment became important. The intake by the drinking water was not important, but due to the high value of $\mathrm{K}_{\mathrm{ow}}$ the products were accumulated in fish. Bennett et al. ${ }^{20}$ suggested exposure via other livestock could similarly be dominant for chemicals with a high Kow and half-life in other media.

Water emissions:

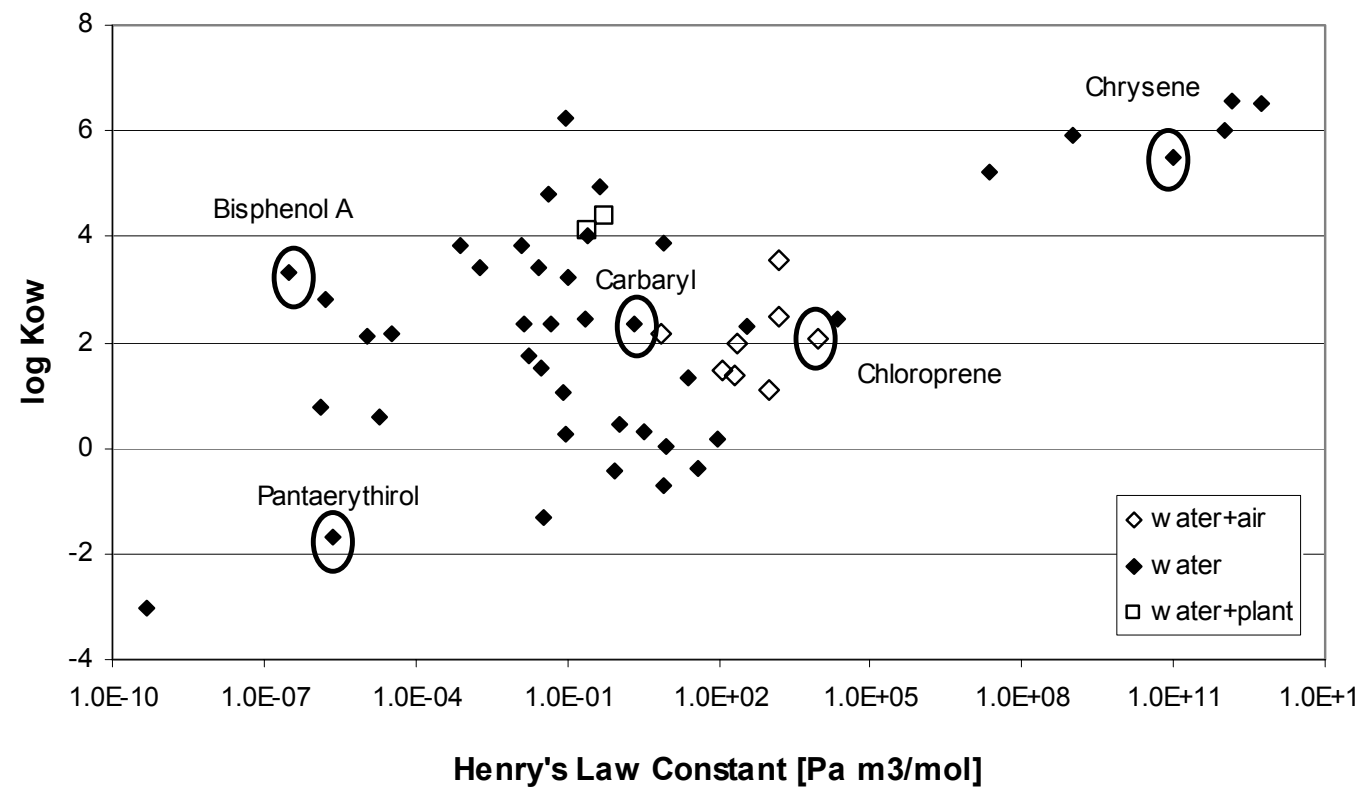

Figure 4: Uniform water emission for 53 organic chemicals plotted as a function of octanol-water partitioning coefficient (Kow) and Henry's Law constant (see Tables 4 and 5). The symbols denote the key environmental medium that contributed to the intake in the model. 
The majority of the intake for chemicals released to water is primarily influenced by the water compartment concentrations, as the compounds can only migrate to the air or to the sediment (direct transfer to soils is not described in the fate model). As the sediments had no influence on the intake in the current model, a possibly significant problem, the only medium of interest other than water was the air.

Chemicals with small values of $\mathrm{H}$, like Bisphenol A or Pantaerythirol, never left the water. Intake was typically dominated by contaminants in drinking water and in fish, although Bennett et al. ${ }^{20}$ suggested that dermal exposure might also be important.

Bisphenol A has a high value of $\mathrm{K}_{\mathrm{ow}}$, but a rather short degradation half-life in water. The intake was dominated by contaminants in drinking water, not by fish. Pantaerythirol has a small value of $\mathrm{K}_{\mathrm{ow}}$, hence was not accumulated in fish. The intake was again dominated by drinking water in this model.

If $\mathrm{H}$ is greater than $1\left[\mathrm{~Pa} \mathrm{~m} \mathrm{~m}^{3} / \mathrm{mol}\right]$ an important fraction of the total mass can leave the water. For some compounds like Chloroprene inhalation was then an important factor in terms of human intake. But a high value of $\mathrm{H}$ alone wasn't sufficient that inhalation became dominant. The chemicals must also have a long degradation half-life in air. Products with a very high $\mathrm{H}$ but low atmospheric half-life, like Chrysene, were still dominated by contaminants in the water in terms of human intake (long degradation half-life in water). In addition, these compounds have a very high value of $\mathrm{K}_{\mathrm{ow}}$ and they were accumulated in fish in the model.

For chemicals like Chlorothalonil, located in the middle of water-dominated compounds in Figure 3, plants also played an important role. This was due to their long half-life in air. Once in the air, these compounds can partition strongly to plants and were accumulated in the plant in the model. The value of $\mathrm{H}$ isn't high enough, however, that the air compartment became the dominant pathway. Bennett et al. ${ }^{20}$ also suggested that dermal exposure could be dominant for chemicals with an intermediate value of Kow, although they questioned these findings and dermal exposure was not addressed here.

Table 4: Half life data of considered chemicals. Selection criteria as per Table 1, although based on a chemical set used by Amman ${ }^{4}$.

\begin{tabular}{|c|c|c|c|c|c|c|c|}
\hline \multirow[t]{2}{*}{ Chemical } & \multicolumn{7}{|c|}{ Half Life Data [hours] } \\
\hline & $\left|\begin{array}{l}\text { troposphe } \\
\text { re }\end{array}\right|$ & $\begin{array}{l}\text { Water } \\
\text { column }\end{array}$ & $\begin{array}{l}\text { sedimen } \\
t \\
\text { (aerobic } \\
\text { ) }\end{array}$ & $\begin{array}{l}\text { vegetatio } \\
\text { n }\end{array}$ & $\begin{array}{l}\text { Soil } \\
\text { surface } \\
\text { layer }\end{array}$ & $\begin{array}{l}\text { Soil root } \\
\text { zone }\end{array}$ & $\begin{array}{l}\text { Soil } \\
\text { vadose } \\
\text { layer }\end{array}$ \\
\hline \multirow[b]{2}{*}{ EPN } & & $5.04 \mathrm{E}+\mathrm{C}$ & $5.04 \mathrm{E}+0$ & $5.00 \mathrm{E}+0$ & $5.04 \mathrm{E}+\mathrm{C}$ & $5.04 \mathrm{E}+0$ & $5.04 \mathrm{E}+0$ \\
\hline & $5.00 \mathrm{E}+00$ & & 2 & 0 & 2 & 2 & 2 \\
\hline \multirow{3}{*}{ free cyanide ion } & & $1.00 \mathrm{E}+\mathrm{c}$ & $1.00 \mathrm{E}+9$ & $1.00 \mathrm{E}+9$ & $1.00 \mathrm{E}+\mathrm{C}$ & $1.00 \mathrm{E}+9$ & $1.00 \mathrm{E}+9$ \\
\hline & $1.28 \mathrm{E}+04$ & & 9 & 9 & & 9 & 9 \\
\hline & & $5.96 \mathrm{E}+\mathrm{C}$ & $5.96 \mathrm{E}+0$ & $1.21 \mathrm{E}+0$ & $5.96 \mathrm{E}+\mathrm{C}$ & $5.96 \mathrm{E}+0$ & $2.38 \mathrm{E}+0$ \\
\hline \multirow[t]{2}{*}{ Fluoranthene } & $1.21 \mathrm{E}+01$ & 3 & 3 & 1 & 3 & 3 & 4 \\
\hline & & $6.32 \mathrm{E}+\mathrm{C}$ & $6.32 \mathrm{E}+0$ & $1.73 \mathrm{E}+0$ & $6.32 \mathrm{E}+\mathrm{C}$ & $6.32 \mathrm{E}$ & $2.53 \mathrm{E}+0$ \\
\hline Benzo[a]anthracene & $1.73 \mathrm{E}+00$ & & 3 & 0 & & 3 & 4 \\
\hline Chrysene & $4.81 \mathrm{E}+00$ & $1.46 \mathrm{E}+\mathrm{C}$ & $1.46 \mathrm{E}+0$ & $4.81 \mathrm{E}+0$ & $1.46 \mathrm{E}+\mathrm{C}$ & $1.46 \mathrm{E}+0$ & $5.85 \mathrm{E}+0$ \\
\hline
\end{tabular}

Supporting Information, Pennington et al. "Spatial versus non-spatial modelling" 


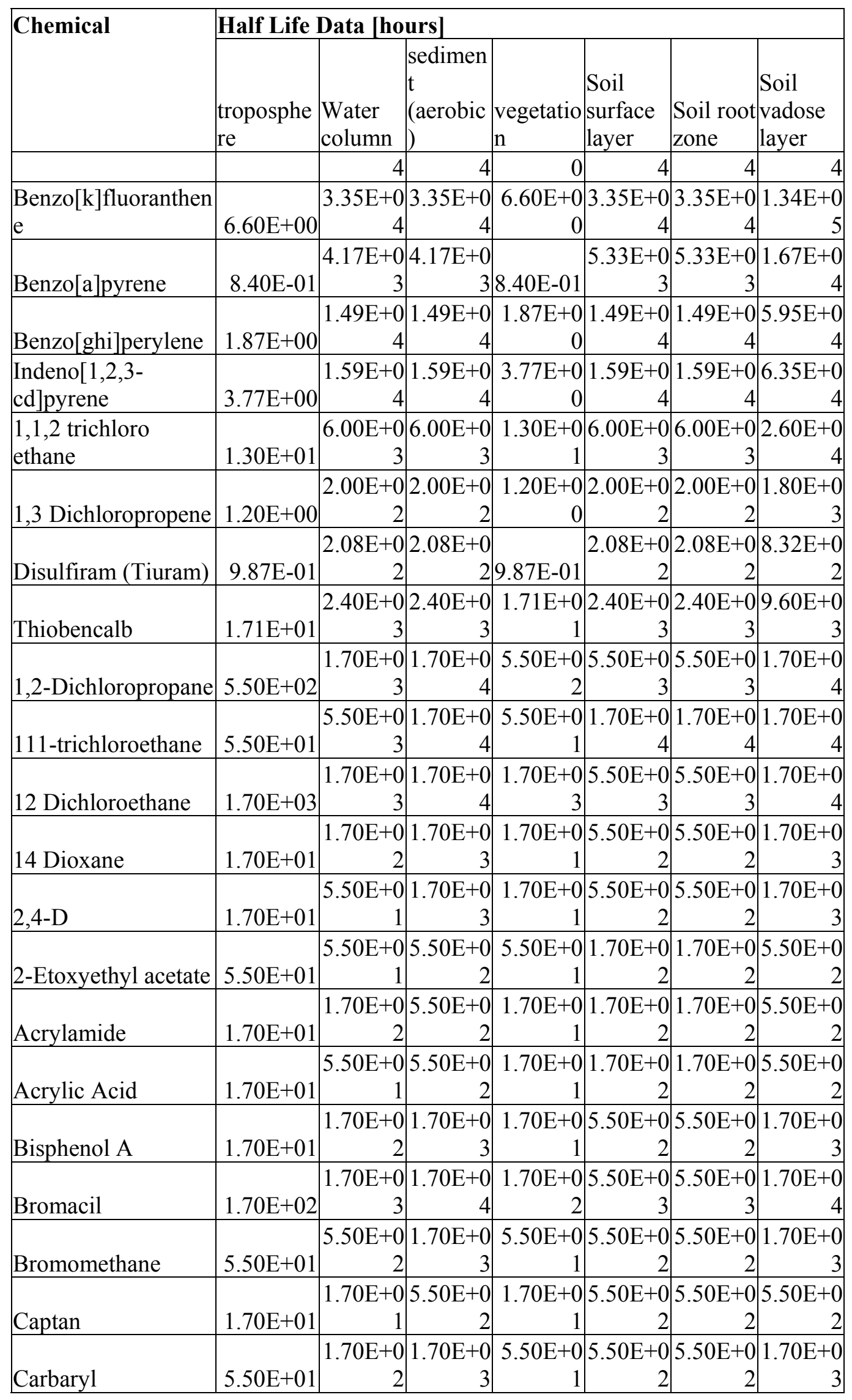

Supporting Information, Pennington et al. "Spatial versus non-spatial modelling" 


\begin{tabular}{|c|c|c|c|c|c|c|c|}
\hline \multirow[t]{2}{*}{ Chemical } & \multicolumn{7}{|c|}{ Half Life Data [hours] } \\
\hline & $\begin{array}{l}\text { troposphe } \\
\text { re }\end{array}$ & \begin{tabular}{|l|} 
Water \\
column
\end{tabular} & $\begin{array}{l}\text { sedimen } \\
t \\
(\text { aerobic }\end{array}$ & vegetatio & $\begin{array}{l}\text { Soil } \\
\text { surface } \\
\text { layer }\end{array}$ & $\begin{array}{l}\text { Soil root } \\
\text { zone }\end{array}$ & t| $\begin{array}{l}\text { Soil } \\
\text { vadose } \\
\text { layer }\end{array}$ \\
\hline \multirow[b]{2}{*}{ Chloroprene } & & $5.50 \mathrm{E}+0$ & $5.50 \mathrm{E}+0$ & $5.50 \mathrm{E}+0$ & $1.70 \mathrm{E}+0$ & $1.70 \mathrm{E}+0$ & $5.50 \mathrm{E}+0$ \\
\hline & $5.50 \mathrm{E}+01$ & 2 & 3 & 1 & 3 & 3 & $3 \quad 3$ \\
\hline \multirow[b]{2}{*}{ Chlorothalonil } & & $1.70 \mathrm{E}+0$ & $1.70 \mathrm{E}+0$ & $1.70 \mathrm{E}+0$ & $5.50 \mathrm{E}+0$ & $5.50 \mathrm{E}+0$ & $1.70 \mathrm{E}+0$ \\
\hline & $1.70 \mathrm{E}+02$ & 2 & 3 & 2 & 2 & 2 & 2 \\
\hline \multirow[b]{2}{*}{ Clorpyrifos } & & $1.70 \mathrm{E}+0$ & $1.70 \mathrm{E}+0$ & $1.70 \mathrm{E}+0$ & $1.70 \mathrm{E}+0$ & $1.70 \mathrm{E}+0$ & $1.70 \mathrm{E}+0$ \\
\hline & $1.70 \mathrm{E}+01$ & 2 & 3 & $3 \quad 1$ & 2 & 2 & $2 \quad 3$ \\
\hline \multirow[b]{2}{*}{ Diazinon } & & $1.70 \mathrm{E}+0$ & $5.50 \mathrm{E}+0$ & $5.50 \mathrm{E}+0$ & $1.70 \mathrm{E}+0$ & $1.70 \mathrm{E}+0$ & $5.50 \mathrm{E}+0$ \\
\hline & $5.50 \mathrm{E}+02$ & 3 & 3 & 2 & 3 & 3 & 3 \\
\hline \multirow[b]{2}{*}{ Dimethoate } & & $5.50 \mathrm{E}+0$ & $5.50 \mathrm{E}+0$ & $5.00 \mathrm{E}+0$ & $1.70 \mathrm{E}+0$ & $1.70 \mathrm{E}+0$ & $5.50 \mathrm{E}+0$ \\
\hline & $5.00 \mathrm{E}+00$ & 2 & 3 & $3 \quad 0$ & 3 & 3 & 3 \\
\hline \multirow[b]{2}{*}{ Dimethylamine } & & $5.50 \mathrm{E}+0$ & $5.50 \mathrm{E}+0$ & $5.00 \mathrm{E}+0$ & $1.70 \mathrm{E}+0$ & $1.70 \mathrm{E}+0$ & $5.50 \mathrm{E}+0$ \\
\hline & $5.00 \mathrm{E}+00$ & 1 & 2 & 0 & 2 & 2 & 2 \\
\hline \multirow[b]{2}{*}{ Diquat Dibromide } & & $5.50 \mathrm{E}+0$ & $1.70 \mathrm{E}+0$ & $1.70 \mathrm{E}+0$ & $5.50 \mathrm{E}+0$ & $5.50 \mathrm{E}+0$ & $1.70 \mathrm{E}+0$ \\
\hline & $1.70 \mathrm{E}+01$ & 1 & 3 & 3 & 2 & 2 & $2 \quad 3$ \\
\hline \multirow[b]{2}{*}{ Disulfoton } & & $1.70 \mathrm{E}+0$ & $1.70 \mathrm{E}+0$ & $5.00 \mathrm{E}+0$ & $5.50 \mathrm{E}+0$ & $5.50 \mathrm{E}+0$ & $1.70 \mathrm{E}+0$ \\
\hline & $5.00 \mathrm{E}+00$ & 1 & 3 & $\begin{array}{l}0 \\
\end{array}$ & 2 & 2 & 2 \\
\hline \multirow[b]{2}{*}{ Endosulfan } & & $1.70 \mathrm{E}+0$ & $1.70 \mathrm{E}+0$ & $1.70 \mathrm{E}+0$ & $5.50 \mathrm{E}+0$ & $5.50 \mathrm{E}+0$ & $1.70 \mathrm{E}+0$ \\
\hline & $1.70 \mathrm{E}+01$ & 2 & 3 & 3 & 2 & 2 & 3 \\
\hline \multirow[b]{2}{*}{ Epichlorohydrin } & & $1.70 \mathrm{E}+0$ & $1.70 \mathrm{E}+0$ & $1.70 \mathrm{E}+0$ & $5.50 \mathrm{E}+0$ & $5.50 \mathrm{E}+0$ & $1.70 \mathrm{E}+0$ \\
\hline & $1.70 \mathrm{E}+01$ & 2 & 3 & 1 & 2 & 2 & 3 \\
\hline \multirow[b]{2}{*}{ EPN - 2} & & $5.50 \mathrm{E}+0$ & $5.50 \mathrm{E}+0$ & $5.50 \mathrm{E}+0$ & $1.70 \mathrm{E}+0$ & $1.70 \mathrm{E}+0$ & $5.50 \mathrm{E}+0$ \\
\hline & $5.50 \mathrm{E}+01$ & 1 & 2 & 1 & 2 & 2 & 2 \\
\hline \multirow[b]{2}{*}{ Ethanol Amine } & & $5.50 \mathrm{E}+0$ & $5.50 \mathrm{E}+0$ & $1.70 \mathrm{E}+0$ & $1.70 \mathrm{E}+0$ & $1.70 \mathrm{E}+0$ & $5.50 \mathrm{E}+0$ \\
\hline & $1.70 \mathrm{E}+01$ & 1 & 2 & 2 & 2 & 2 & 2 \\
\hline \multirow[b]{2}{*}{ Fenthion } & & $1.70 \mathrm{E}+0$ & $1.70 \mathrm{E}+0$ & $1.70 \mathrm{E}+0$ & $5.50 \mathrm{E}+0$ & $5.50 \mathrm{E}+0$ & $1.70 \mathrm{E}+0$ \\
\hline & $1.70 \mathrm{E}+02$ & 3 & 4 & $4 \quad 2$ & 3 & 3 & 3 \\
\hline \multirow[b]{2}{*}{ Isoprene } & & $1.70 \mathrm{E}+0$ & $1.70 \mathrm{E}+0$ & $5.00 \mathrm{E}+0$ & $5.50 \mathrm{E}+0$ & $5.50 \mathrm{E}+0$ & $1.70 \mathrm{E}+0$ \\
\hline & $5.00 \mathrm{E}+00$ & 2 & 3 & \begin{tabular}{|l|}
0 \\
\end{tabular} & 2 & 2 & 3 \\
\hline \multirow[b]{2}{*}{ Malathion } & & $5.50 \mathrm{E}+0$ & $5.50 \mathrm{E}+0$ & $1.70 \mathrm{E}+0$ & $5.50 \mathrm{E}+0$ & $5.50 \mathrm{E}+0$ & $5.50 \mathrm{E}+0$ \\
\hline & $1.70 \mathrm{E}+01$ & 1 & 2 & 1 & 1 & 1 & 12 \\
\hline \multirow[b]{2}{*}{ Methidathion } & & $5.50 \mathrm{E}+0$ & $5.50 \mathrm{E}+0$ & $5.50 \mathrm{E}+0$ & $1.70 \mathrm{E}+0$ & $1.70 \mathrm{E}+0$ & $5.50 \mathrm{E}+0$ \\
\hline & $5.50 \mathrm{E}+01$ & 1 & 2 & 1 & 2 & 2 & $2 \quad 2$ \\
\hline \multirow[b]{2}{*}{ Methomyl } & & $5.50 \mathrm{E}+0$ & $5.50 \mathrm{E}+0$ & $5.50 \mathrm{E}+0$ & $5.50 \mathrm{E}+0$ & $5.50 \mathrm{E}+0$ & $5.50 \mathrm{E}+0$ \\
\hline & $5.50 \mathrm{E}+02$ & 3 & 3 & $3 \quad 2$ & 2 & 2 & $2 \quad 3$ \\
\hline \multirow[b]{2}{*}{ Molinate } & & $5.50 \mathrm{E}+0$ & $5.50 \mathrm{E}+0$ & $1.70 \mathrm{E}+0$ & $1.70 \mathrm{E}+0$ & $1.70 \mathrm{E}+0$ & $5.50 \mathrm{E}+0$ \\
\hline & $1.70 \mathrm{E}+01$ & 1 & 2 & 2 & 2 & 2 & 2 \\
\hline \multirow[b]{2}{*}{ Momomethylamine } & & $5.50 \mathrm{E}+0$ & $5.50 \mathrm{E}+0$ & $5.00 \mathrm{E}+0$ & $1.70 \mathrm{E}+0$ & $1.70 \mathrm{E}+0$ & $5.50 \mathrm{E}+0$ \\
\hline & $5.00 \mathrm{E}+00$ & 1 & 2 & 0 & 2 & 2 & $2 \quad 2$ \\
\hline \multirow[b]{2}{*}{ Pentaerythritol } & & $5.50 \mathrm{E}+0$ & $5.50 \mathrm{E}+0$ & $5.50 \mathrm{E}+0$ & $1.70 \mathrm{E}+0$ & $1.70 \mathrm{E}+0$ & $5.50 \mathrm{E}+0$ \\
\hline & $5.50 \mathrm{E}+01$ & 1 & 2 & 1 & 2 & 2 & 2 \\
\hline & & $5.50 \mathrm{E}+0$ & $5.50 \mathrm{E}+0$ & $5.50 \mathrm{E}+0$ & $1.70 \mathrm{E}+0$ & $1.70 \mathrm{E}+0$ & $5.50 \mathrm{E}+0$ \\
\hline Pronamide & $5.50 \mathrm{E}+01$ & 1 & 2 & 2 & 2 & 2 & 2 \\
\hline Propoxur & $5.00 \mathrm{E}+00$ & $5.50 \mathrm{E}+0$ & $1.70 \mathrm{E}+0$ & $5.00 \mathrm{E}+0$ & $5.50 \mathrm{E}+0$ & $5.50 \mathrm{E}+0$ & $1.70 \mathrm{E}+0$ \\
\hline
\end{tabular}

Supporting Information, Pennington et al. "Spatial versus non-spatial modelling" 


\begin{tabular}{|c|c|c|c|c|c|c|c|}
\hline \multirow[t]{2}{*}{ Chemical } & \multicolumn{7}{|c|}{ Half Life Data [hours] } \\
\hline & $\begin{array}{l}\text { troposphe } \\
\text { re }\end{array}$ & \begin{tabular}{|l|} 
\\
Water \\
column
\end{tabular} & $\begin{array}{l}\text { sedimen } \\
\mathrm{t} \\
(\text { aerobic } \\
\text { ) }\end{array}$ & $\begin{array}{l}\text { vegetatio } \\
\text { n }\end{array}$ & $\begin{array}{l}\text { Soil } \\
\text { surface } \\
\text { layer }\end{array}$ & $\begin{array}{l}\text { Soil root } \\
\text { zone }\end{array}$ & $\begin{array}{l}\text { Soil } \\
\text { tadose } \\
\text { layer }\end{array}$ \\
\hline & & 2 & 3 & 0 & $0 \quad 2$ & 2 & 2 \\
\hline \multirow[b]{2}{*}{ Propylene Oxide } & & $1.70 \mathrm{E}+0$ & $1.70 \mathrm{E}+0$ & $1.70 \mathrm{E}+0$ & $05.50 \mathrm{E}+0$ & $5.50 \mathrm{E}+0$ & $1.70 \mathrm{E}+0$ \\
\hline & $1.70 \mathrm{E}+03$ & 2 & 3 & 3 & $3 \quad 2$ & 2 & 2 \\
\hline \multirow[b]{2}{*}{ Simazine - MacLeod } & & $5.50 \mathrm{E}+0$ & $5.50 \mathrm{E}+0$ & $5.50 \mathrm{E}+0$ & $01.70 \mathrm{E}+0$ & $1.70 \mathrm{E}+0$ & $5.50 \mathrm{E}+0$ \\
\hline & $5.50 \mathrm{E}+01$ & 2 & 3 & 1 & 13 & $3 \quad 3$ & $3 \quad 3$ \\
\hline \multirow[b]{2}{*}{ Thiuram } & & $1.70 \mathrm{E}+0$ & $1.70 \mathrm{E}+0$ & $1.70 \mathrm{E}+0$ & $05.50 \mathrm{E}+0$ & $5.50 \mathrm{E}+0$ & $1.70 \mathrm{E}+0$ \\
\hline & $1.70 \mathrm{E}+02$ & 2 & 3 & 2 & $2 \quad 2$ & 2 & 2 \\
\hline \multirow[b]{2}{*}{ 13Dichloropropene } & & $1.70 \mathrm{E}+0$ & $1.70 \mathrm{E}+0$ & $5.50 \mathrm{E}+0$ & $05.50 \mathrm{E}+0$ & $5.50 \mathrm{E}+0$ & $1.70 \mathrm{E}+0$ \\
\hline & $5.50 \mathrm{E}+01$ & 2 & 3 & 1 & $1 \quad 2$ & 2 & $2 \quad 3$ \\
\hline \multirow[b]{2}{*}{ Ethyl Acrylate } & & $5.50 \mathrm{E}+0$ & $5.50 \mathrm{E}+0$ & $1.70 \mathrm{E}+0$ & $01.70 \mathrm{E}+0$ & $1.70 \mathrm{E}+0$ & $5.50 \mathrm{E}+0$ \\
\hline & $1.70 \mathrm{E}+01$ & 1 & 2 & 1 & $1 \quad 2$ & 2 & 2 \\
\hline \multirow[b]{2}{*}{ 112-Trichloroethane } & & $1.70 \mathrm{E}+0$ & $1.70 \mathrm{E}+0$ & $5.50 \mathrm{E}+0$ & $05.50 \mathrm{E}+0$ & $5.50 \mathrm{E}+0$ & $1.70 \mathrm{E}+0$ \\
\hline & $5.50 \mathrm{E}+03$ & 3 & 4 & 3 & $\begin{array}{l}3 \\
\end{array}$ & $3 \quad 3$ & \begin{tabular}{l|l}
3 & 4 \\
\end{tabular} \\
\hline \multirow[b]{2}{*}{ TCDD } & & $1.03 \mathrm{E}+0$ & $4.94 \mathrm{E}+0$ & $5.23 \mathrm{E}+0$ & $05.23 \mathrm{E}+0$ & $1.60 \mathrm{E}+0$ & $1.60 \mathrm{E}+0$ \\
\hline & $7.20 \mathrm{E}+02$ & 4 & 4 & 4 & $4 \quad 4$ & $4 \quad 5$ & $5 \quad 5$ \\
\hline
\end{tabular}

Table 5: Physical properties of considered chemicals (see Table 4).

\begin{tabular}{|c|c|c|c|c|c|c|}
\hline Chemical & \begin{tabular}{|l|} 
Molecular \\
Mass \\
(g/mole) \\
\end{tabular} & $\begin{array}{l}\text { Henry's } \\
\text { Constant } \\
\text { (Pa m3 } \\
\text { mol-1) }\end{array}$ & Log Kow Chemical & \begin{tabular}{|l|} 
Molecular \\
Mass \\
(g/mole)
\end{tabular} & \begin{tabular}{|l|} 
Henry's \\
Constant \\
(Pa m3 \\
mol-1)
\end{tabular} & $\log K$ \\
\hline EPN & $3.23 \mathrm{E}+02$ & $1.30 \mathrm{E}-02$ & $3.85 \mathrm{E}+00$ Clorpyrifos & $3.51 \mathrm{E}+02$ & $4.38 \mathrm{E}-01$ & $4.96 \mathrm{E}$ \\
\hline free cyanide ion & $2.60 \mathrm{E}+01$ & $2.42 \mathrm{E}+03$ & -6.90E-01 Diazinon & $3.04 \mathrm{E}+02$ & $7.38 \mathrm{E}-04$ & $3.81 \mathrm{E}$ \\
\hline Fluoranthene & $2.02 \mathrm{E}+02$ & $2.25 \mathrm{E}+07$ & $5.22 \mathrm{E}+00$ Dimethoate & $2.29 \mathrm{E}+02$ & $1.38 \mathrm{E}-06$ & $7.90 \mathrm{E}$ \\
\hline Benzo[a]anthracene & $2.28 \mathrm{E}+02$ & $9.78 \mathrm{E}+08$ & 5.91E+00 Dimethylamine & $4.51 \mathrm{E}+01$ & $3.92 \mathrm{E}+01$ & $-3.80 \mathrm{E}$ \\
\hline Chrysene & $2.28 \mathrm{E}+02$ & $1.02 \mathrm{E}+11$ & 5.50E+00 Diquat Dibromide & $3.44 \mathrm{E}+02$ & $4.92 \mathrm{E}-10$ & $-3.05 \mathrm{E}$ \\
\hline Benzo[k]fluoranthene & $2.52 \mathrm{E}+02$ & $1.05 \mathrm{E}+12$ & $6.00 \mathrm{E}+00$ Disulfoton & $2.74 \mathrm{E}+02$ & $2.63 \mathrm{E}-01$ & $4.02 \mathrm{E}$ \\
\hline Benzo[a]pyrene & $2.52 \mathrm{E}+02$ & $9.20 \mathrm{E}-02$ & $6.26 \mathrm{E}+00$ Endosulfan & $4.07 \mathrm{E}+02$ & $1.53 \mathrm{E}+03$ & $3.55 \mathrm{E}$ \\
\hline Benzo[ghi]perylene & $2.68 \mathrm{E}+02$ & $5.31 \mathrm{E}+12$ & $6.50 \mathrm{E}+00$ Epichlorohydrin & $9.25 \mathrm{E}+01$ & $3.29 \mathrm{E}+00$ & $3.00 \mathrm{E}$ \\
\hline Indeno[1,2,3-cd]pyrene & $2.76 \mathrm{E}+02$ & $1.45 \mathrm{E}+12$ & $6.58 \mathrm{E}+00 \mathrm{EPN}-2$ & $3.23 \mathrm{E}+02$ & $4.51 \mathrm{E}-02$ & $4.78 \mathrm{E}$ \\
\hline $1,1,2$ trichloro ethane & $1.33 \mathrm{E}+02$ & $1.21 \mathrm{E}+02$ & $1.48 \mathrm{E}+00$ Ethanol Amine & $6.11 \mathrm{E}+01$ & $3.24 \mathrm{E}-02$ & $-1.31 \mathrm{E}$ \\
\hline 1,3 Dichloropropene & $1.11 \mathrm{E}+02$ & $3.80 \mathrm{E}+02$ & $2.28 \mathrm{E}+00$ Fenthion & $2.78 \mathrm{E}+02$ & $2.65 \mathrm{E}-01$ & $4.09 \mathrm{E}$ \\
\hline Disulfiram (Tiuram) & $2.97 \mathrm{E}+02$ & $8.30 \mathrm{E}+00$ & $3.88 \mathrm{E}+00$ Isoprene & $1.19 \mathrm{E}+02$ & $2.14 \mathrm{E}+04$ & $2.42 \mathrm{E}$ \\
\hline Thiobencalb & $2.58 \mathrm{E}+02$ & $2.67 \mathrm{E}-02$ & 3.40E+00 Malathion & $3.30 \mathrm{E}+02$ & $1.35 \mathrm{E}-02$ & $2.36 \mathrm{E}$ \\
\hline 1,2-Dichloropropane & $1.13 \mathrm{E}+02$ & $2.32 \mathrm{E}+02$ & 1.99E+00Methidathion & $3.02 \mathrm{E}+02$ & $2.34 \mathrm{E}-01$ & $2.42 \mathrm{E}$ \\
\hline 111-trichloroethane & $1.33 \mathrm{E}+02$ & $1.55 \mathrm{E}+03$ & 2.49E+00Methomyl & $1.62 \mathrm{E}+02$ & $1.86 \mathrm{E}-05$ & $6.00 \mathrm{E}$ \\
\hline 12 Dichloroethane & $9.90 \mathrm{E}+01$ & $9.18 \mathrm{E}+01$ & 1.70E-01 Molinate & $1.22 \mathrm{E}+02$ & $1.03 \mathrm{E}-01$ & $3.21 \mathrm{E}$ \\
\hline 14 Dioxane & $8.81 \mathrm{E}+01$ & $8.69 \mathrm{E}-01$ & -4.20E-01 Momomethylamine & $3.11 \mathrm{E}+01$ & $8.35 \mathrm{E}+00$ & $-7.10 \mathrm{E}$ \\
\hline 2,4-D & $1.37 \mathrm{E}+02$ & $1.69 \mathrm{E}-06$ & $2.81 \mathrm{E}+00$ Pentaerythritol & $1.36 \mathrm{E}+02$ & $2.27 \mathrm{E}-06$ & $-1.69 \mathrm{E}$ \\
\hline 2-Etoxyethyl acetate & $1.32 \mathrm{E}+02$ & $9.19 \mathrm{E}-02$ & 2.40E-01 Pronamide & $2.56 \mathrm{E}+02$ & $1.93 \mathrm{E}-03$ & $3.43 \mathrm{E}$ \\
\hline Acrylamide & $7.11 \mathrm{E}+01$ & $8.27 \mathrm{E}-02$ & $1.04 \mathrm{E}+00$ Propoxur & $2.09 \mathrm{E}+02$ & $3.14 \mathrm{E}-02$ & $1.52 \mathrm{E}$ \\
\hline
\end{tabular}




\begin{tabular}{|c|c|c|c|}
\hline Acrylic Acid & 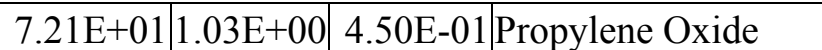 & $5.81 \mathrm{E}+01 \mid 9.31 \mathrm{E}+00$ & $3.00 \mathrm{E}$ \\
\hline Bisphenol A & \begin{tabular}{l|ll}
$2.28 \mathrm{E}+02$ & $3.11 \mathrm{E}-07$ & $3.32 \mathrm{E}+00$ \\
\end{tabular} & \begin{tabular}{l|l|}
$2.02 \mathrm{E}+02$ & $3.27 \mathrm{E}-05$ \\
\end{tabular} & $2.18 \mathrm{E}$ \\
\hline Bromacil & \begin{tabular}{|l|l|l|l|}
$2.61 \mathrm{E}+02$ & $1.06 \mathrm{E}-05$ & $2.11 \mathrm{E}+00$ & Thiuram \\
\end{tabular} & \begin{tabular}{|l|l|}
$2.40 \mathrm{E}+02$ & $1.84 \mathrm{E}-02$ \\
\end{tabular} & $1.73 \mathrm{E}$ \\
\hline Bromomethane & \begin{tabular}{ll|l|l|l|l}
$9.49 \mathrm{E}+01$ & $9.99 \mathrm{E}+02$ & $1.08 \mathrm{E}+00$ & 13 Dichloropropene
\end{tabular} & \begin{tabular}{l|l|l|}
$1.11 \mathrm{E}+02$ & $2.14 \mathrm{E}+02$ \\
\end{tabular} & $1.36 \mathrm{E}$ \\
\hline Captan & \begin{tabular}{l|l|l|}
$1.26 \mathrm{E}+02$ & $4.96 \mathrm{E}-02$ & $2.35 \mathrm{E}+00$ Ethyl Acrylate \\
\end{tabular} & $1.00 \mathrm{E}+022.54 \mathrm{E}+01$ & $1.32 \mathrm{E}$ \\
\hline Carbaryl & \begin{tabular}{l|l|l|l|l}
$1.27 \mathrm{E}+02$ & $2.22 \mathrm{E}+00$ & $2.34 \mathrm{E}+00$ & 112 -Trichloroethane
\end{tabular} & \begin{tabular}{l|l|}
$1.33 \mathrm{E}+02$ & $7.51 \mathrm{E}+00$ \\
\end{tabular} & $2.17 \mathrm{E}$ \\
\hline Chloroprene & 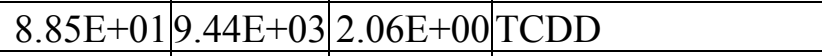 & \begin{tabular}{l|l|l|}
$3.22 \mathrm{E}+02$ & $2.47 \mathrm{E}+00$ \\
\end{tabular} & $6.64 \mathrm{E}$ \\
\hline Chlorothalonil & \begin{tabular}{l|l|l|l|}
$2.66 \mathrm{E}+02$ & $5.76 \mathrm{E}-01$ & $4.38 \mathrm{E}+00$ & \\
\end{tabular} & & \\
\hline
\end{tabular}

\section{Differences between predictions and monitoring observations}

A number of potential reasons exist for the differences between model predictions using estimated emissions data and observations based on monitoring data, including:

- Air measurements are often made close to the surface, whereas the height of the atmospheric boundary layer in the model was fixed at $800 \mathrm{~m}$ with an assumed uniform concentration distribution in this layer. Measured concentrations at low levels, particularly in locations close to sources, may be higher than the more regional-scale model averages. Additionally, the average mixing height varies as a function of location and time.

- Concentrations in the root soil were underestimated by about 2 orders of magnitude. The soil-air volatilization rate was predicted to be higher than the degradation rate, hence controlling the overall residence time in the soil. The reliability of such estimates requires further investigation. Additionally the average concentration in the $30 \mathrm{~cm}$ depth of modeled root soil is compared to monitoring sample data from a 2 to $10 \mathrm{~cm}$ depth. Thirdly, monitoring samples may not be from remote locations relative to point emission sources or represent averages in watersheds.

- The model currently performs calculations in a steady state mode to provide long-term fate and exposure insights. As efforts have been made to reduce the emissions of dioxins, the emission profile has changed over the years. Since PeCDF is a persistent chemical (overall half-life in the model of 24 years, with most of the mass in soils) the monitored concentrations may be influenced by historical emissions and, hence, higher than the model estimates using current emissions data.

\section{River Flow rates Comparison}

For each zone, the mean annual run off was determined based a $0.5 \times 0.5$ degree grid. For each grid cell the mean annual run off (rainfall - evapotranspiration) is given. The data were provided from the Global Runoff Data Centre (GRDC) $)^{21}$. Based on the run off, the discharge of every zone was calculated using the following expression:

$$
Q_{\text {out }}=\text { area }^{*} R O_{\text {mean }}
$$

where $\mathrm{Q}_{\text {out }}=$ flow rate out of the zone, area $=$ surface of the zone and $\mathrm{RO}_{\text {mean }}=$ mean run off.

Supporting Information, Pennington et al. "Spatial versus non-spatial modelling" 
In Table 6, the computed flow rates of five major rivers are compared to the measured data. There are small differences. Compared to other uncertainties in the model, this precession was considered sufficient. The applied method also has the advantage that all the calculated flow rates are based on the same data.

Table 6: Comparison of measured ${ }^{21}$ and computed river flow rates.

\begin{tabular}{|l|l|l|}
\hline River & $\mathbf{Q}_{\text {measured }}\left[\mathbf{m}^{\mathbf{3}} / \mathbf{s}\right]$ & $\mathbf{Q}_{\text {calculated }}\left[\mathbf{m}^{\mathbf{3}} / \mathbf{s}\right]$ \\
\hline Rhein & 2278 & 2386.09 \\
\hline Rhône & 1693 & 1662.01 \\
\hline Po & 1515 & 1325.22 \\
\hline Loire & 838 & 841.47 \\
\hline Ebro & 483 & 526.28 \\
\hline
\end{tabular}

\section{Modeled Environmental Compartments}

The compartments taken into account in each watershed and grid cell are illustrated in Figure 5. Spatial differentiation was not taken into account within each of the watersheds or grid cells. Western Europe was modeled with both a single air compartment in the non-spatial version of the model, as well as multiple air compartments using a grid. Oceanic waters were similarly modeled using the grid cells, each with a sediment and two water layers. Each watershed was considered to consist of a soil, a surface water, a sediment compartment, and an agricultural vegetation compartment.

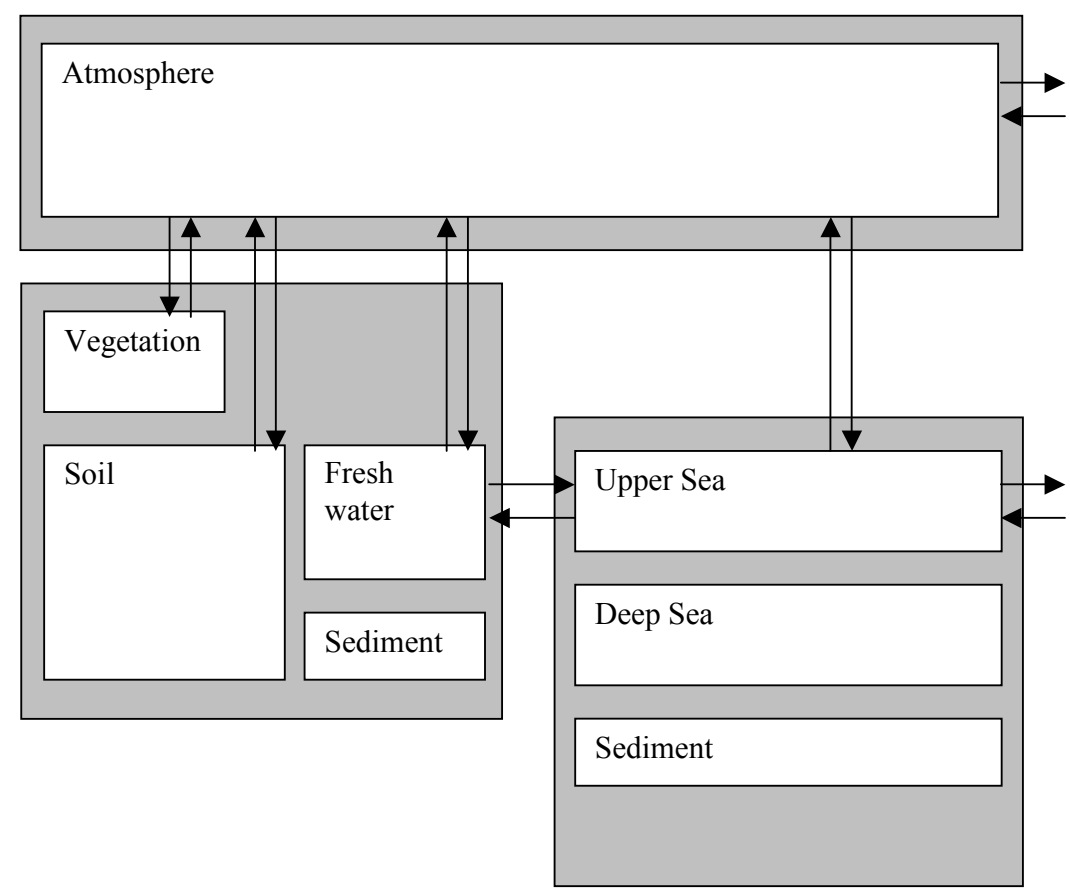

Figure 5: Illustration of the environmental compartments taken into account, distributed according to atmospheric grid cells, watersheds, and oceanic grid cells. 


\section{Use of watershed versus grid delimitations for atmospheric modeling}

Pelichet ${ }^{5}$ presented a detailed analysis of the implications in multimedia models of using a grid-based delimitation versus using the watershed boundaries as delimitations for the atmospheric compartments. In both cases, bi-directional advection flow rates between air compartments were estimated using the average annual wind velocity data and from the perpendicular cross-sectional areas for each delimitation option (see Table 1 in paper).

The watershed delimitation for air compartments suggests higher dispersion and dilution for some chemicals compared to using the grid-based delimitation. Figure 6 presents a straightforward hypothetical situation to highlight this issue. Figure 6 takes into account the full advective flow data (north, south, east, and west bi-directional velocities). This discrepancy is of lower relevance than presented when modeling emissions that are uniformly dispersed across Western Europe, rather than location specific emissions.

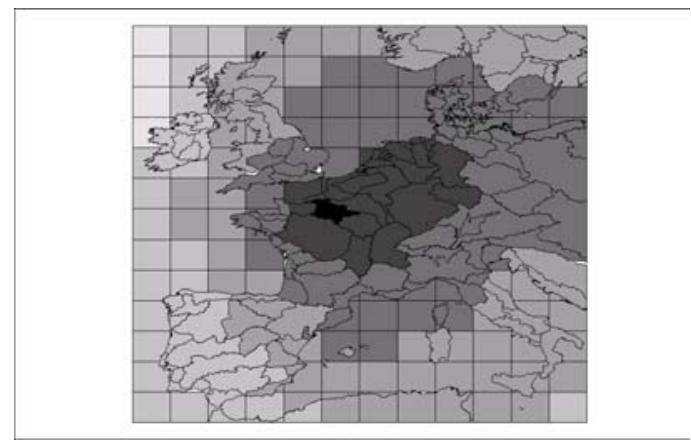

a) watershed delimited

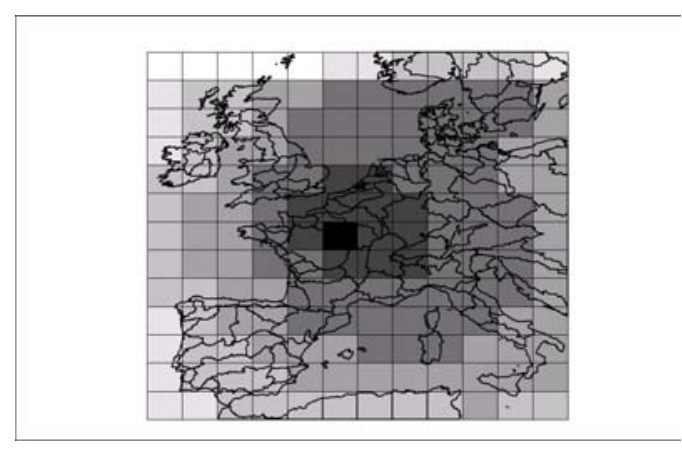

b) grid delimited

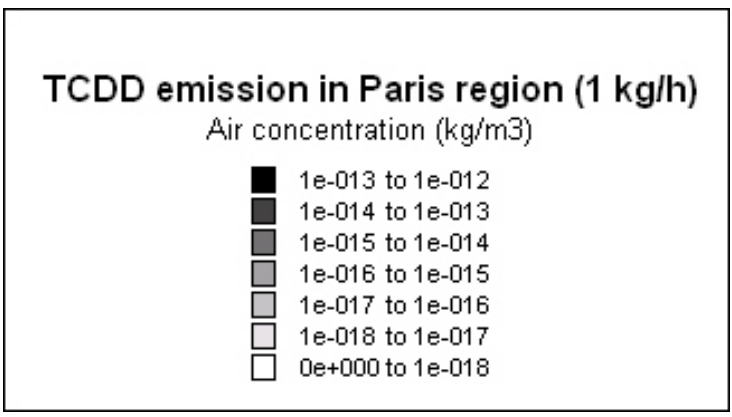

Figure 6: Comparison of watershed versus grid air advection model delimitations for a fictive TCDD emission in Northern France using the complete multimedia model with all bi-directional atmospheric advection patterns.

\section{List of Lakes and Lake Parameters}

Table 7: Lakes and data that were taken into account in the model

\begin{tabular}{|c|c|c|}
\hline Lake & Volume $\left[\mathrm{m}^{3}\right]$ & Mean depth [m] \\
\hline Tjeukemeer $^{+}$ & $4.20 \mathrm{E}+07$ & 2 \\
\hline Attersee $^{+}$ & $3.95 \mathrm{E}+09$ & 84.2 \\
\hline Lake Maggiore $^{+}$ & $3.75 \mathrm{E}+10$ & 176.5 \\
\hline
\end{tabular}

Supporting Information, Pennington et al. "Spatial versus non-spatial modelling" 


\begin{tabular}{|c|c|c|}
\hline Lake Zurich $^{+}$ & $3.30 \mathrm{E}+09$ & 51 \\
\hline Lake Geneva $^{+}$ & $8.89 \mathrm{E}+10$ & 152.7 \\
\hline Loch Ness $^{+}$ & $7.45 \mathrm{E}+08$ & 132 \\
\hline Lake Lunzer $^{+}$ & $1.30 \mathrm{E}+07$ & 20 \\
\hline Windermere $^{+}$ & $3.15 \mathrm{E}+08$ & 21.3 \\
\hline Lake Ree $^{+}$ & $6.51 \mathrm{E}+08$ & 6.2 \\
\hline Lake Derg $^{+}$ & $8.87 \mathrm{E}+08$ & 7.6 \\
\hline Ammernsee $^{+}$ & $1.77 \mathrm{E}+09$ & 38.1 \\
\hline Stammberger See $^{+}$ & $3.00 \mathrm{E}+09$ & 21.2 \\
\hline Loch Morar $^{+}$ & $2.30 \mathrm{E}+09$ & 86.6 \\
\hline Loch Shiel $^{+}$ & $9.00 \mathrm{E}+08$ & 40.5 \\
\hline Loch Awe $^{+}$ & $1.20 \mathrm{E}+09$ & 2.7 \\
\hline Loch Lomonnd $^{+}$ & $2.60 \mathrm{E}+09$ & 2 \\
\hline Lake Trasimeno $^{+}$ & $5.86 \mathrm{E}+08$ & 4.7 \\
\hline Lake Stechlin $^{+}$ & $9.69 \mathrm{E}+07$ & 22.8 \\
\hline Lake Constance $^{+}$ & $4.85 \mathrm{E}+10$ & 90 \\
\hline Lough Neagh $^{+}$ & $3.45 \mathrm{E}+09$ & 8.9 \\
\hline Lake Slapy $^{+}$ & $2.70 \mathrm{E}+07$ & 20.7 \\
\hline Lake Annecy $^{+}$ & $1.12 \mathrm{E}+09$ & 41.5 \\
\hline Lake Orta $^{+}$ & $1.24 \mathrm{E}+09$ & 70.9 \\
\hline Lake Banyeles $^{+}$ & $1.61 \mathrm{E}+07$ & 14.8 \\
\hline Lago di Garda $^{+}$ & $4.90 \mathrm{E}+10$ & 136 \\
\hline Emmerdale $^{+}$ & $5.33 \mathrm{E}+07$ & 17.8 \\
\hline Derwentwater $^{+}$ & $2.90 \mathrm{E}+07$ & 5.5 \\
\hline Crummock $^{+}$ & $6.64 \mathrm{E}+07$ & 26.7 \\
\hline Coniston $^{+}$ & $1.13 \mathrm{E}+08$ & 24.1 \\
\hline Lago di Como $^{+}$ & $2.25 \mathrm{E}+10$ & 153 \\
\hline Bergummermeer $^{+}$ & $6.00 \mathrm{E}+09$ & 1.3 \\
\hline Bassenthwaite $^{+}$ & $2.79 \mathrm{E}+07$ & 5.3 \\
\hline Arendsee $^{+}$ & $1.47 \mathrm{E}+08$ & 28.6 \\
\hline Haweswater $^{+}$ & $7.66 \mathrm{E}+07$ & 23.4 \\
\hline Lago di Lugano $^{+}$ & $4.40 \mathrm{E}+09$ & 160 \\
\hline Wast Water $^{+}$ & $1.16 \mathrm{E}+08$ & 39.7 \\
\hline Lake Zug $^{+}$ & $3.18 \mathrm{E}+09$ & 14.2 \\
\hline Ullswater $^{+}$ & $2.23 \mathrm{E}+08$ & 25.3 \\
\hline Lake of Neuchâtel* & $1.42 \mathrm{E}+11$ & 64.8 \\
\hline Lake of Thun* & $6.50 \mathrm{E}+09$ & 134 \\
\hline Lake of Bienne* & $1.24 \mathrm{E}+09$ & 31.2 \\
\hline Lake of Brienz* & $5.17 \mathrm{E}+09$ & 173 \\
\hline Lake of Walen* & $2.49 \mathrm{E}+09$ & 103 \\
\hline Lake of Morrat* & $6.00 \mathrm{E}+08$ & 26.1 \\
\hline Lake of Lucerne & $1.18 \mathrm{E}+10$ & 104 \\
\hline Traunsee $^{+}$ & $2.23 \mathrm{E}+09$ & 91.3 \\
\hline
\end{tabular}

* Data from BWG ${ }^{22} \quad{ }^{+}$Date from ILEC ${ }^{23}$ 


\section{Non-Spatial Version Model Parameters (also used in spatial version where spatial resolution was not taken into account, as stated in the paper)}

Table 8: Default parameters in the air module

\begin{tabular}{|l|l|}
\hline $\begin{array}{l}\text { water-side diffusion mass transfer coeff }(\mathrm{kw}-\mathrm{m} / \mathrm{h}) \text { - for air-water } \\
\text { diffusion }\end{array}$ & 0.05 \\
\hline air-side diffusion mass transfer coeff $(\mathrm{ka}-\mathrm{m} / \mathrm{h})$ - for air-water diffusion & 5 \\
\hline dry deposition velocity $(\mathrm{m} / \mathrm{h})$ & $1.00 \mathrm{E}+01$ \\
\hline wet deposition scavenging volume $(\mathrm{m} 3 \mathrm{air} / \mathrm{m} 3 \mathrm{rain})$ & $2.00 \mathrm{E}+05$ \\
\hline air boundary layer thickness $(\mathrm{m})$ & 0.005 \\
\hline Lower to upper atmospheric loss rate const. $(\mathrm{m} / \mathrm{h})$ & $1.00 \mathrm{E}-04$ \\
\hline temp $(\mathrm{K})$ & 298 \\
\hline Aerosol solid density $(\mathrm{kg} / \mathrm{m} 3)$ & 2400 \\
\hline Height of atmospheric boundary layer $(\mathrm{m})$ & 800 \\
\hline Aerosol phase fraction $(\mathrm{m} 3 / \mathrm{m} 3$ air) & $2.00 \mathrm{E}-11$ \\
\hline fraction of soil covered by vegetation & 0.8 \\
\hline Fraction of rain intercepted by foliage $($ FrUF) & 0.1 \\
\hline Leaf Area Index $(\mathrm{LAI})(\mathrm{m} 2 / \mathrm{m} 2)$ & 4 \\
\hline air side air-veg. Mass transfer boundary layer thickness $(\mathrm{m})$ & 0.002 \\
\hline fraction of water in Vegetation & 0.75 \\
\hline fraction of (psuedo) octanol in Veg & 0.02 \\
\hline Surface soil Layer & \\
\hline volumetric water fraction & 0.3 \\
\hline volumetric air fraction & 0.2 \\
\hline volumetric solids fraction & 0.5 \\
\hline & \\
\hline
\end{tabular}

Table 9: Default parameters of the soil module

\begin{tabular}{|l|l|}
\hline ppm of solids in runoff water & 200 \\
\hline solid phase soil density $(\mathrm{kg} / \mathrm{m} 3)$ & 2400 \\
\hline fraction of organic carbon in solid phase of soil & 0.02 \\
\hline temp (K) & 298 \\
\hline air boundary layer thickness (m) & 0.005 \\
\hline fraction of soil covered by vegetation & 0.8 \\
\hline Surface Layer & \\
\hline Depth (m) & 0.01 \\
\hline volumetric water fraction & 0.3 \\
\hline volumetric air fraction & 0.2 \\
\hline volumetric solids fraction & 0.5 \\
\hline Root Zone & \\
\hline Depth (m) & 0.8 \\
\hline volumetric water fraction & 0.3 \\
\hline volumetric air fraction & 0.2 \\
\hline volumetric solids fraction & 0.5 \\
\hline Vadose Layer & \\
\hline Depth (m) & 1.5 \\
\hline
\end{tabular}

Supporting Information, Pennington et al. "Spatial versus non-spatial modelling" 


\begin{tabular}{|l|l|}
\hline volumetric water fraction & 0.3 \\
\hline volumetric air fraction & 0.2 \\
\hline volumetric solids fraction & 0.5 \\
\hline
\end{tabular}

Infiltration: A default value of 0.8 was applied for the infiltration. This default value is widely used for land improvement (drainage, irrigation) in the absence of measured data. Note that the infiltration factor is only applicable for soils. Impermeable/paved area was modelled separately.

Table 10: Default parameters of the vegetation module

\begin{tabular}{|l|l|}
\hline fraction of soil covered by vegetation & 0.8 \\
\hline Leaf Area Index (LAI) (m2/m2) & 4 \\
\hline Veg mass per square meter of soil (kg/m2) & 1 \\
\hline fraction of water in Vegetation & 0.75 \\
\hline fraction of (psuedo) octanol in Veg & 0.02 \\
\hline air side air-veg. Mass transfer boundary layer thickness (m) & 0.002 \\
\hline Fraction of rain intercepted by foliage (FrUF) & 0.1 \\
\hline Average Vegetation cycle (days) & 240 \\
\hline vegetation solids density (kg/m3) & 600 \\
\hline temp (K) & 298 \\
\hline
\end{tabular}

Table 11: Default parameters of the water module

\begin{tabular}{|l|l|}
\hline water $\mathrm{pH}$ & 7 \\
\hline suspended sediment density $(\mathrm{kg} / \mathrm{m} 3)$ & 1500 \\
\hline fraction of organic carbon in solid phase of sus. sed. & 0.2 \\
\hline sediment deposition rate constant $(\mathrm{m} / \mathrm{h})$ & $5.00 \mathrm{E}-07$ \\
\hline Suspended sediment Phase fraction $(\mathrm{m} 3 / \mathrm{m} 3$ water $)$ & $5.00 \mathrm{E}-06$ \\
\hline temp $(\mathrm{K})$ & 298 \\
\hline $\begin{array}{l}\text { water-side diffusion mass transfer coeff }(\mathrm{kw}-\mathrm{m} / \mathrm{h}) \quad \text { - for air-water } \\
\text { diffusion }\end{array}$ & 0.05 \\
\hline \begin{tabular}{l} 
air-side diffusion mass transfer coeff $(\mathrm{ka}-\mathrm{m} / \mathrm{h})-$ for air-water diffusion \\
\hline sediment to water mass transfer coefficient $(\mathrm{m} / \mathrm{h})$
\end{tabular} & 5 \\
\hline & $1.00 \mathrm{E}-04$ \\
\hline
\end{tabular}

Table 12: Default parameters of the sedimentation module

\begin{tabular}{|l|l|}
\hline sediment to water mass transfer coefficient $(\mathrm{m} / \mathrm{h})$ & $1.00 \mathrm{E}-04$ \\
\hline sediment deposition rate constant $(\mathrm{m} / \mathrm{h})$ & $5.00 \mathrm{E}-07$ \\
\hline fraction of sediment deposition buried & 0.4 \\
\hline fraction of sediment deposition resuspended & 0.4 \\
\hline solid phase sediment density $(\mathrm{kg} / \mathrm{m} 3)$ & 2400 \\
\hline fraction of organic carbon in solid phase of sediment & 0.04 \\
\hline sediment depth $(\mathrm{m})$ & 0.05 \\
\hline solids fraction & 0.2 \\
\hline
\end{tabular}

Table 13: Default parameters of the sea module

\begin{tabular}{|l|l|}
\hline depth of upper layer $(\mathrm{m})$ & 10 \\
\hline Suspended sediment Phase fraction - top & $5.00 \mathrm{E}-06$ \\
\hline depth of lower layer $(\mathrm{m})$ & 90 \\
\hline
\end{tabular}

Supporting Information, Pennington et al. "Spatial versus non-spatial modelling" 


\begin{tabular}{|l|l|}
\hline Suspended sediment Phase fraction - bottom & $5.00 \mathrm{E}-06$ \\
\hline water PH & 7 \\
\hline suspended sediment density $(\mathrm{kg} / \mathrm{m} 3)$ & $1.50 \mathrm{E}+03$ \\
\hline fraction of organic carbon in sus. sed. & 0.2 \\
\hline vertical mixing velocity $(\mathrm{m} / \mathrm{h})$ & $1.00 \mathrm{E}-03$ \\
\hline air-side diffusion mass transfer coeff $(\mathrm{ka}-\mathrm{m} / \mathrm{h})$ & $5.00 \mathrm{E}+00$ \\
\hline water-side diffusion mass transfer coeff $(\mathrm{kw}-\mathrm{m} / \mathrm{h})$ & $5.00 \mathrm{E}-02$ \\
\hline temp $(\mathrm{K})$ & $2.98 \mathrm{E}+02$ \\
\hline sediment deposition rate constant $(\mathrm{m} / \mathrm{h})$ & $5.00 \mathrm{E}-07$ \\
\hline sediment to water mass transfer coefficient $(\mathrm{m} / \mathrm{h})$ & $1.00 \mathrm{E}-04$ \\
\hline
\end{tabular}

\section{Algebraic Chemical Fate Modelling Mathematical Solutions}

This section presents an illustrative example of how to establish a first order mass balance for the four compartment chemical fate model in Figure 7.

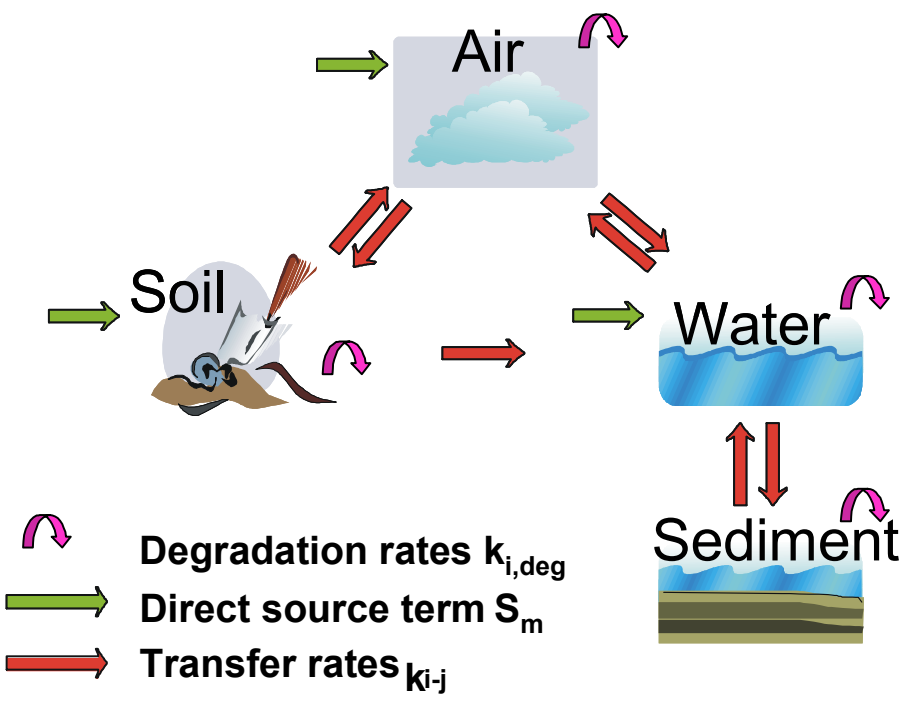

Figure 7: Example for a four compartments system. (Indices: $\mathrm{i}-\mathrm{a}$ compartment, $\mathrm{j}-$ another compartment, deg - degradation, $\mathrm{m}$ - emission compartment.)

Mass balance equations:

$$
\begin{aligned}
& \frac{d M_{a}}{d t}=-M_{a} \cdot k_{a t o t}+M_{s} \cdot k_{s-a}+M_{w} \cdot k_{w-a}+S_{a}, \text { where } k_{a t o t}=k_{a \mathrm{deg}}+k_{a-s}+k_{a-w} \\
& \frac{d M_{w}}{d t}=-M_{w} \cdot k_{w t o t}+M_{a} \cdot k_{a-w}+M_{s} \cdot k_{s-w}+M_{d} \cdot k_{d-w}+S_{w}, \text { where } \\
& k_{w t o t}=k_{w \mathrm{deg}}+k_{w-d}+k_{w-a} \\
& \frac{d M_{s}}{d t}=-M_{s} \cdot k_{s t o t}+M_{a} \cdot k_{a-s}+S_{s}, \text { where } k_{s t o t}=k_{s \mathrm{deg}}+k_{s-w}+k_{s-a} \\
& \frac{d M_{d}}{d t}=-M_{d} \cdot k_{d t o t}+M_{w} \cdot k_{w-d}, \text { where } k_{d t o t}=k_{d \mathrm{deg}}+k_{d-w}
\end{aligned}
$$

Supporting Information, Pennington et al. "Spatial versus non-spatial modelling” 
where:

$\mathrm{M}_{\mathrm{i}}$ : $\quad$ mass in medium $\mathrm{i}[\mathrm{kg}]$;

$\mathrm{k}_{\mathrm{m}-\mathrm{n}}: \quad$ transfer rate from medium $\mathrm{m}$ to medium $\mathrm{n}$ [1/day];

$\mathrm{k}_{\mathrm{tot}}$ : denotes total removal rate coefficient, the sum of all the removal coefficients

Indices: a - air; w - water; s - soil; d - sediment; deg - degradation; tot - total (sum of all loss processes);

For this four compartment system under consideration the same equations can be written as:

$$
\begin{gathered}
\left(\begin{array}{l}
d M_{a} / d t \\
d M_{w} / d t \\
d M_{s} / d t \\
d M_{d} / d t
\end{array}\right)=\left(\begin{array}{cccc}
-k_{a t o t} & k_{w-a} & k_{s-a} & 0 \\
k_{a-w} & -k_{w t o t} & k_{s-w} & k_{d-w} \\
k_{a-s} & 0 & -k_{s t o t} & 0 \\
0 & k_{w-d} & 0 & -k_{d t o t}
\end{array}\right) \cdot\left(\begin{array}{c}
M_{a} \\
M_{w} \\
M_{s} \\
M_{d}
\end{array}\right)+\left(\begin{array}{c}
S_{a} \\
S_{w} \\
S_{s} \\
0
\end{array}\right) \\
\dot{M}(t)=\quad-\bar{M}+\hat{S}
\end{gathered}
$$

Using this matrix approach, iterative calculations are unnecessary and difficult-to-spot errors are reduced when compared to using other algebraic techniques. Run times are minimized, 2 minutes being required to run the current spatial model. The matrix of rate coefficients additionally provides a transparent basis for gaining quick insights into the relative importance of advective transport, intermedia transport, as well as the degradation/other losses from each compartment, region, or medium in the spatial model. A rate coefficient in the matrix is essentially the inverse of the half-life $(\mathrm{k}=$ $\left.\ln (2) / \tau_{1 / 2}\right)$.

\section{Fate Rate Coefficient Algorithm Summary}

For organic chemicals, for the fate calculations, the rate coefficients for intermedia transport were mainly based on generic algorithms summarized below. These are generally similar to those adopted in other multimedia models and were partly selected through a review of these commonly adopted approaches ${ }^{1}$.

- Schwarzenbach et al. ${ }^{24}$ for intermedia transfer coefficients from surface waters for diffusion and sedimentation

- Mackay et al. ${ }^{25}$ for diffusion and resuspension from sediments

- Mackay et al. ${ }^{25}$ for diffusion, dry and wet deposition and dissolution from air

- Finizio et al. ${ }^{26}$ for transfer to particulate matter from the gas phase within air

- Hauschild \& Jolliet ${ }^{27}$ for corrections to account for the intermittent nature of rainfall events in the air on wet deposition and dissolution in steady-state models

- McKone \& Bennett ${ }^{28}$ to estimate the vertical chemical profile of a chemical in soil due to advection through the soil, diffusion, and degradation 
- McKone \& Bennett ${ }^{28}$ and Mackay et al. ${ }^{25}$ for transfers from soils via diffusion and for advection/diffusive transfer into the saturated vadose layer.

- Charles $^{3}$ and Cousins \& Mackay ${ }^{29}$ for transfers to/from agricultural vegetation via diffusion, root/stem uptake from soils, and through deposition onto foliage surfaces.

The following sections list all the specific rate coefficients that were taken into account. The rate coefficients for the oceanic water modules and deep sea sediments are analogous to those for freshwater bodies.

The encoded algorithms can be accessed in the IMPACT 2002 model Excel spreadsheets (available at www.epfl.ch/impact), as well as the cited sources. In all cases these were calculated on a bulk concentration basis, accounting for equilibrium phase partitioning within media as outlined in Mackay et al. ${ }^{25}$. Cross-checks were made with other models that adopt these algorithms, such as CalTox ${ }^{12}$, for quality assurance purposes. Further details of the equations are available in the literature cited above and in a separate summary document available from the authors for this model.

\section{1) Freshwater compartment rate coefficients}

$\mathrm{k}_{\mathrm{w}-\mathrm{a}}-$ Transfer rate coefficient from fresh water to air by diffusion [1/day];

$\mathrm{k}_{\mathrm{w}-\mathrm{d}}$ - Transfer rate coefficient from fresh water to sediment by diffusion and sedimentation [1/day];

$\mathrm{k}_{\mathrm{w}, \text { advzi-zj }}$ - Advective transfer rate coefficient out of the fresh water compartment $\mathrm{i}$ into fresh water of $\mathrm{j}[1 /$ day $]$;

$\mathrm{k}_{\mathrm{wdeg}}-$ Bulk degradation rate coefficient in fresh water [1/day];

\section{2) Freshwater sediment surface layer rate coefficients}

$\mathrm{k}_{\mathrm{d}-\mathrm{w}}$ - Transfer rate coefficient from fresh water sediment to fresh water by diffusion and re-suspension [1/day];

$\mathrm{k}_{\mathrm{ddeg}}$ - Bulk degradation rate in fresh water sediment [1/day];

$\mathrm{k}_{\text {burial }}$ - Burial loss rate in fresh water sediment [1/day];

\section{3) Rate coefficients for vegetation}

$\mathrm{k}_{\mathrm{sr}-1}$ - Transfer rate from surface residue to leaf [1/day];

$\mathrm{k}_{\text {srdeg }}$ - Degradation rate in plant surface residue [1/day];

$\mathrm{k}_{\text {sr,ha }}$ - Harvesting loss rate of plant surface residue [1/day];

$\mathrm{k}_{1-\mathrm{a}}$ - Diffusion transfer rate from leaf to air [1/day];

$\mathrm{k}_{1-\mathrm{sr}}$ - Transfer rate from leaf to surface residue [1/day];

$\mathrm{k}_{1-\mathrm{st}}$ - Transfer rate from leaf to stem [1/day];

$\mathrm{k}_{\text {ldeg }}$ - Degradation rate in leaf [1/day];

$\mathrm{k}_{1, \mathrm{ha}}$ - Harvesting loss rate of leaf [1/day];

$\mathrm{k}_{\mathrm{st}-1}-$ Transfer rate from stem to leaf [1/day];

Supporting Information, Pennington et al. "Spatial versus non-spatial modelling" 
$\mathrm{k}_{\mathrm{stdeg}}$ - Degradation rate in the stem [1/day];

$\mathrm{k}_{\mathrm{st}, \mathrm{ha}}$ - Harvesting loss rate of stem [1/day];

$\mathrm{K}_{\text {stts }}$ - Stem-transpiration stream equilibrium distribution coefficient [-];

\section{4) Atmospheric rate coefficients}

$\mathrm{k}_{\mathrm{a}-\mathrm{w}}-$ Transfer rate coefficient from air to surface water by diffusion, dry ( $\mathrm{k}_{\mathrm{a}-}$ w,dry $)$ and wet $\left(\mathrm{k}_{\mathrm{a}-\mathrm{w}, \mathrm{wet}}\right)$ deposition, and dissolution [1/day];

$\mathrm{k}_{\mathrm{a}-\mathrm{s}}$ - Transfer rate coefficient from air to soil by diffusion, dry $\left(\mathrm{k}_{\mathrm{a}-\mathrm{s} \text {,dry }}\right)$ and wet $\left(\mathrm{k}_{\mathrm{a}-\mathrm{s}, \mathrm{wet}}\right)$ deposition, and dissolution [1/day];

$\mathrm{k}_{\mathrm{a}-1}-$ Transfer rate coefficient from air to plant leaf by diffusion, dry $\left(\mathrm{k}_{\mathrm{a}-1, \mathrm{l} d r y}\right)$ and wet $\left(\mathrm{k}_{\mathrm{a}-\mathrm{l}, \mathrm{wet}}\right)$ deposition, and dissolution [1/day];

$\mathrm{k}_{\mathrm{adry}, \text { tot }}$ - Total transfer rate coefficient from air during dry period [1/day];

$\mathrm{k}_{\text {adeg }}$ - Bulk degradation in air [1/day];

\section{5) Transfers from soil}

$\mathrm{k}_{\mathrm{s}-\mathrm{w}}$ - Transfer rate coefficient from surface soil to fresh water by runoff (solids and aqueous) [1/day];

$\mathrm{k}_{\mathrm{s}-\mathrm{w}}$ - Transfer rate coefficient from surface soil to fair by diffusion [1/day];

$\mathrm{k}_{\mathrm{s}-\mathrm{st}}-$ Transfer rate coefficient from root soil to plant stems/roots [1/day];

$\mathrm{k}_{\mathrm{s}-\mathrm{v}}$ - Transfer rate coefficient from unsaturated vadose layer in soils to saturated vadose layer by diffusion and advection [1/day];

\section{Exposure Rate Coefficients}

Further detailed discussions of the exposure data and the model calculations are available in Margni ${ }^{2}$. Algorithms are also encoded in the model IMPACT 2002 Excel spreadsheet (available at www.epfl.ch/impact).

Substance dependent properties:

Chemical properties:

$\mathrm{K}_{\mathrm{ow}}$ - Octanol-water partitioning coefficient;

$\mathrm{BTF}_{\mathrm{e}}-$ Biotransfer factor for substrate e [day $/ \mathrm{kg}_{\mathrm{e}}$ wet weight];

$\mathrm{BAF}_{\mathrm{ei}}-$ Bioaccumulation factor in exposure substrate e from a contaminated environmental medium $\mathrm{i}\left[\mathrm{kg}_{\text {chemical }} \cdot \mathrm{kg}_{\mathrm{e}}^{-1}\right.$ wet weight per $\mathrm{kg}_{\text {chemical }} \cdot \mathrm{kg}_{\mathrm{i}}{ }^{-1}$ wet weight];

\section{Intermediate parameters:}

$\mathrm{E}_{\mathrm{i}, \text { direct }}$ - Direct intake rate of medium i $\left[\mathrm{m}_{\mathrm{i}}^{3} /\right.$ day $]$;

$E_{i, \text { indirect }}-$ Equivalent indirect intake rate associated with the medium $i$ via exposure route $\mathrm{xr}\left[\mathrm{m}^{3}{ }_{\mathrm{i}} /\right.$ day $]$;

$\mathrm{M}_{\mathrm{i}}-$ Chemical mass in medium i $\left[\mathrm{kg}_{\text {chemical }}\right]$;

Substance independent properties:

$f_{\text {fat }, e}-$ Fat content of substrate e [-];

Supporting Information, Pennington et al. "Spatial versus non-spatial modelling" 
$b_{e}-$ Coefficient of BTF-regression specific to exposure substrate e $(e=$ milk, meat and eggs) [-];

$\mathrm{E}_{\mathrm{i}}{ }^{\prime}$ - Specific farm animal feed intake of medium $\mathrm{i}$ in fresh-mass for pigs, poultry, and goats \& sheep, respectively $\left[\mathrm{kg}_{\mathrm{i}} / \mathrm{day}\right]$;

$\mathrm{E}_{\mathrm{e}}$ - Intake rate of substrate e $\left[\mathrm{kg}_{\mathrm{e}} / \mathrm{day}\right]$;

$\mathrm{P}_{\mathrm{e}}$ - Production rate of bulk exposure substrate e (or food produce like meat, milk, eggs and fish) $\left[\mathrm{kg}_{\mathrm{e}} / \mathrm{day}\right]$;

$\mathrm{P}_{\mathrm{i}}$ - Production or extraction rate of bulk medium i, i.e. quantity of medium $\mathrm{i}$ taken from environment (or spatial cell) $\left[\mathrm{kg}_{\mathrm{i}} / \mathrm{day}\right]$;

F - Fraction of usable/edible production/substrate or medium [-];

$\rho_{\mathrm{i}}-$ Bulk density of medium i $\left[\mathrm{kg}_{\mathrm{i}} / \mathrm{m}_{\mathrm{i}}^{3}\right]$;

$\mathrm{V}_{\mathrm{i}}$ - Medium bulk volume $\left[\mathrm{m}^{3}\right]$

$\mathrm{n}_{\mathrm{xp}}-$ Number of exposure pathways [-];

$\mathrm{i}$ - Medium index (considered media are: air, surface water, exposed produce, unexposed produce, agricultural soil (accidentally)); as opposed to a substrate indexed by 'e' a medium is part of the environmental fate model;

e - Exposure substrate index (considered exposure substrates are: fresh water fish, sea water fish, pig meat, beef meat, poultry meat, goat \& sheep meat, eggs, milk) ; as opposed to a medium indexed by ' $\mathrm{i}$ ' a substrate is not part of the environmental fate model;

$\mathrm{z}$ - Spatial zone;

\section{Drinking water:}

The chemical fraction adsorbed to particulate matter was assumed to be eliminated in the purification/filtration process for surface waters ${ }^{30}$, although the sensitivity of the results to this assumption was low in this model.

\section{Estimating bioaccumulation factors for organic compounds}

\section{Terrestrial farm animals:}

Bioaccumulation factors (BAF) from one specific medium to a given exposure substrate can by default be estimated by multiplying predicted biotransfer factors (BTF) by farm animal intake rates:

$$
B A F_{e i}=B T F_{e} \cdot E_{i}^{\prime}
$$

where $E_{\mathrm{i}}$ ' denotes the individual farm animal species intake rate, such as daily mass of vegetation, water, soil or air consumed by the respective food producing animal (see the following Table).

Table 14: Mean values of farm animal intake $\left(\mathrm{E}_{\mathrm{i}}{ }^{\prime}\right)$ and meat fat content by species. Roughage fresh mass (FM) is obtained assuming leaf water content of 0.73 . Industrial feed of plant origin and non-vegetal origin are directly given in FM.

Supporting Information, Pennington et al. "Spatial versus non-spatial modelling" 


\begin{tabular}{|c|c|c|c|c|c|c|c|}
\hline \multirow{4}{*}{$\begin{array}{l}\text { Animal farm } \\
\text { species }\end{array}$} & \multicolumn{6}{|c|}{ Individual farm animal intake rate $\mathrm{E}_{\mathrm{i}}{ }^{\prime}$} & \multirow[b]{3}{*}{$\begin{array}{l}\text { Meat fat } \\
\text { content }\end{array}$} \\
\hline & \multirow[b]{2}{*}{$\begin{array}{l}\text { Roughage } \\
\mathrm{M}_{\text {leaves }}\end{array}$} & \multicolumn{2}{|c|}{ Industrial feed } & \multirow[b]{2}{*}{$\begin{array}{l}\text { Water } \\
\mathrm{M}_{\mathrm{w}}{ }^{*} \rho_{\mathrm{w}}\end{array}$} & \multirow[b]{2}{*}{$\begin{array}{l}\text { Soil } \\
\mathrm{M}_{\mathrm{sa}}\end{array}$} & \multirow[b]{2}{*}{$\begin{array}{l}\text { Air } \\
M_{a}^{*} \rho_{a}\end{array}$} & \\
\hline & & $\begin{array}{l}\begin{array}{l}\text { Plant } \\
\text { origin }\end{array} \\
\mathrm{M}_{\text {stem }}{ }^{2}\end{array}$ & $\begin{array}{l}\text { Non- } \\
\text { veg. } \\
\text { Origin }{ }^{1} \\
\text { Madditives }\end{array}$ & & & & \\
\hline & $\mathrm{kg}_{\mathrm{FM}} /$ day & $\mathrm{kg}_{\mathrm{FM}} / \mathrm{da}$ & $\mathrm{kg}_{\mathrm{FM}} / \mathrm{da}$ & 1/day & $\mathrm{kg} /$ day & $\mathrm{m}^{3} /$ day & unitless \\
\hline Dairy cattle & 56.0 & 3.21 & 0.21 & 60 & 0.8 & 122 & $\mathrm{n} / \mathrm{a}$ \\
\hline $\begin{array}{l}\text { Beef cattle (incl. } \\
\text { calves+veal) }\end{array}$ & 25.8 & 0.83 & 0.05 & 30 & 0.3 & 80 & 0.25 \\
\hline Pigs & 7.7 & 1.15 & 0.11 & 7 & 0.04 & 60 & 0.23 \\
\hline Laying hens & 0.12 & 0.14 & 0.01 & 0.1 & 0.002 & 2.2 & $\mathrm{n} / \mathrm{a}$ \\
\hline Poultry & 0.35 & 0.02 & 0.003 & 0.1 & 0.001 & 2.2 & 0.06 \\
\hline Sheep and goat & 4.6 & 0.17 & 0.008 & 7 & 0.1 & 60 & 0.14 \\
\hline
\end{tabular}

If no measured $\mathrm{BTF}_{\mathrm{e}}$ for meat, milk and eggs is available, it is derived by:

$$
\begin{aligned}
\log B T F_{e} & =\log K_{o w}-b_{e} & \text { if } \log K_{o w}-b_{e}<-1 \\
\log B T F_{e} & =-1 & \text { if } \log K_{o w}-b_{e} \geq-1
\end{aligned}
$$

where $b_{e}$ depends on the exposure substrate e. The values for $b_{e}$ can be found in the table below. The upper limit of -1 (in log scale) is adopted according to Bennett et al. $^{20}$.

Table 15: Coefficients for biotransfer factor calculations.

\begin{tabular}{lll}
\hline $\begin{array}{c}\text { Exposure substrate } \\
\text { (e) }\end{array}$ & $\mathbf{b}_{\mathbf{e}}$ \\
\hline eggs & $5.1^{3}$ & \\
milk & $8.1^{4}$ \\
meat $^{5}$ & $5.6-\log \frac{f_{\text {fat,e }}}{E_{i}^{\prime}}$ where $f_{\text {fat,e }}$ (dimensionless) is the meat fat \\
\hline
\end{tabular}

\footnotetext{
${ }^{1}$ This category is not considered as a contamination source for animal produce in model, because presently it is too difficult to estimate its origin

${ }^{2}$ As industrial feed from plant origin is mainly made by grain (wheat, corn,...) and this latter is associated to unexposed produce, we assume the stem compartment being representative for industrial feed from plant origin.

${ }^{3}$ Given by McKone T. E. 1993. The Precision of QSAE Methods for Estimating Intermedia Transfer Factors in Exposure Assessments. SAR and QSAR in Environmental Research, 1, 41-51.

${ }^{4}$ Given by Travis C. and Arms A. 1988. Bioconcentration of organics in beef, milk, and vegetation. Environ. Sci. Technol., 22, 271-274.

${ }^{5} b_{e}$ for meat is modified from Travis and Arms (1998) coefficient regression accounting for beef fat content and feed intake rate, i.e. $b=7.6-\log (26 / 0.25)$, as suggested in Margni, M. 2003 Source to Intake Modelling in Life Cycle Impact Assessment, Doctoral Thesis, Swiss Federal Institute of Technology (EPFL), Lausanne, Switzerland.
} 
content of a given farm animal species and $E_{\mathrm{i}}^{\prime}\left(\mathrm{kg}_{\mathrm{i}} / \mathrm{day}\right)$ its specific feed intake in fresh-mass for cows, pigs, poultry, and goats \& sheep, respectively (see table below)

Fish:

Measured $\mathrm{BCF}$ for fish are adopted in priority. If no measured $\mathrm{BCF}$ is available for fish, then the BCF is calculated based on the improved method of Meylan et al ${ }^{31}$. The method is encoded in BCFW in software, which gives directly the estimated values.

\section{Food production rate modifications for livestock feeds}

When the produced pasture or industrial feed for farm animals produced in a given region is exported to another region, one has to adjust $\mathrm{P}_{\mathrm{e}}$ accounting for the exposure substrate production that the exported pasture ultimately supports. This can be calculated as follows:

$$
\mathrm{P}_{e}=P_{i, z o n e} \cdot \frac{\mathrm{P}_{\mathrm{e}, \mathrm{EU}}}{\mathrm{P}_{\mathrm{i}, \mathrm{EU}}}
$$

where the ratio $\mathrm{P}_{\mathrm{e}, \mathrm{EU}} / \mathrm{P}_{\mathrm{i}, \mathrm{EU}}\left(\mathrm{kg}_{\mathrm{e}} / \mathrm{day}\right) /\left(\mathrm{kg}_{\mathrm{i}} / \mathrm{day}\right)$ determines the mean European exposure substrate production supported by the industrial feed production. It is assumed to be linearly proportional to the industrial feed production of each spatial cell. Thus, the meat production rate $\mathrm{P}_{\mathrm{e}}$ can be determined knowing the amount of feed production from a given spatial cell, $\mathrm{P}_{\mathrm{i} \text {,zone }}\left(\mathrm{kg}_{\mathrm{i}, \mathrm{zone}} / \mathrm{day}\right)$ and the mean exposure substrate production $\mathrm{P}_{\mathrm{e}}$ (milk, meat, egg) is supported by a given amount of pasture.

\section{References for Supporting Information}

(1) Pennington, D. Prototype Chemical Fate and Human Exposure Model for Japan's National Life Cycle Assessment Initiative, Japan Environmental Management Association for Industry, 2001.

(2) Margni, M. Source to Intake Modeling in Life Cycle Impact Assessment, Doctoral Thesis

N 2773 Swiss Federal Institute of Technology (EPFL), Lausanne, 2003.

(3) Charles, R. Modelling pesticides residues, Doctoral Thesis, Swiss Federal Institute of Technology, Lausanne (EPFL), 2004.

(4) Ammann, C. Development of a spatial multimedia model of chemical fate and human exposure for Europe, Diplome Thesis, Swiss Federal Institute of Technology, Lausanne (EPFL), 2002.

(5) Pelichet, T. Development of a spatial multimedia chemical fate and human exposure model for Europe, with a focus on air advection, Diplome Thesis. Mémoire No. 82 Université de Genève, Lausanne, 2003.

(6) US EPA Notice of Availability of Draft RCRA Waste Minimization PBT Chemical List, Federal Register, EPA 530-Z-98-009, 1998.

(7) Mackay, D.; Shiu, W. Y.; Ma, K. C. Illustrated Handbook of Physical-Chemical

Properties and Environmental Fate for Organic Chemicals; Lewis Publishers: Boca Raton, 1995; Vol. 1-5.

(8) Howard, P. H.; Boethling, R. S.; Jarvis, W. F.; Meylan, W. M.; Michalenko, E. M., Eds. Handbook of Environmental Degradation Rates; Lewis Publishers: Chelsea, MI, 1991.

(9) Howard, P. H. Handbook of Environmental Fate and Exposure Data; Lewis Publishers: Chelsea, MI, 1991; Vol. I-V. 
(10) Syracuse Research Corporation PhysProp chemical property database, http://www.syrres.com/esc/ 2002.

(11) Howard, P.; Meylan, W.; Boethling, R. EPIWIN Suite, http://www.syrres.com/esc/ 2002.

(12) McKone, T.; Bennett, D.; Maddalana, R. CalTOX 4.0 Technical Support Document, Vol. 1, Lawrence Berkeley National Laboratory, 2001.

(13) Huijbregts, M. A. J.; Thissen, U.; Guinee, J. B.; Jager, T.; Kalf, D.; van de Meent, D.; Ragas, A. M. J.; Wegener Sleeswijk, A.; Reijnders, L. Priority assessment of toxic substances in life cycle assessment. Part I: Calculation of Toxicity potentials for 181 substances with the nested multi-media fate, exposure and effects model USES-LCA; Chemosphere 2000, 41, 541-573.

(14) Margni, M.; Pennington, D.; Birkved, M.; Larsen, H. F.; Hauschild, M. Test set of organic chemicals for LCIA characterisation method comparison. Contribution to Workpackage 7 of the OMNIITOX Project, Swiss Federal Institute of Technology (EPFL), Technical University of Denmark, 2002.

(15) Sinkkonen, S.; Paasivirta, J. Degradation half-life times of PCDDs, PCDFs and PCBs for environmental fate modeling.; Chemosphere 2000, 40, 943-949.

(16) McLachlan, M. S.; Thoma, H.; and-others. PCDD/F in an Agricultural Food Chain. Part 1: PCDD/F Mass Balance of a Lactating Cow; Chemosphere 1990, 20, 1013-1020.

(17) Pacyna, J. M. Appendix 1 to executive final summary report. Environmental cycling of selected persistent organic pollutants (POPs) in the Baltic region (Popcycling-Baltic project). $1990 \mathrm{http}: / /$ www.msceast.org/pops/emission.html.

(18) Mackay, D.; Shiu, W. Y.; Ma, K. C. Illustrated Handbook of Physical-Chemical Properties and Environmental Fate for Organic Chemicals; Lewis Publishers: Boca Raton, 1995; Vol. 1-4.

(19) Gouin, T.; MacKay, D.; Webster, E.; Wania, F. Screening for Persistence in the Environment; Environmental Science and Technology 2000, 34, 881-884.

(20) Bennett, D. H.; Margni, M.; McKone, T. E.; Jolliet, O. Intake Fraction for Multimedia Pollutants: A Tool for Life Cycle Analysis and Comparative Risk Assessment; Risk Analysis 2002, 22, 903-916.

(21) Global Runoff Data Centre World Runoff Data, 2002 www.grdc.sr.unh.edu/ 2002.

(22) Bundesamt für Wasser und Geologie Naturseen der Schweitz (Natural lakes of Switzerland), 1983.

(23) International Lake Environment Committee Survey of the State of World Lakes, 2002 http://www.ilec.or.jp/ 2002.

(24) Schwarzenbach, R. P.; Gschwend, P. M.; Imboden, D. M. Environmental Organic Chemistry; Wiley: New York, 1993.

(25) Mackay, D.; Di Guardo, A.; Patterson, S.; Kicsi, G.; Cowan, C. E. Evaluating the environmental fate of a variety of types of chemicals using the EQC model; Environmental Toxicology \& Chemistry 1996, 15, 1627-1637.

(26) Finizio, A.; Mackay, D.; Bidleman, T.; Harner, T. Octanol-air partition coefficient as a predictor of partitioning of semi-volatile organic chemicals to aerosols; Atmospheric Environment 1997, 31, 2289-2296.

(27) Jolliet, O.; Hauschild, M. Determination of atmospheric residence times and transfer factors from air to soil and surface water; submitted manuscript 2003.

(28) McKone, T.; Bennett, D. Algorithms for Estimating Air-Soil Exchange and Penetration Depth in Multimedia Models; Environ. Sci. Technol. 2003, in press.

(29) Cousins, I. T.; Mackay, D. Strategies for including vegetation compartments in multimedia models; Chemosphere, 44, 643-654.

(30) European Commission EUSES, the European Union System for the Evaluation of Substances, National Institute of Public Health and the Environment (RIVM), 1996.

(31) Meylan, W. M.; Howard, P. H.; Boethling, R. S.; Aronson, D.; Printup, H.; Gouchie, S. Improved method for estimating bioconcentration/bioaccumulation factor from octanol/water partition coefficient; Environmental Toxicology and Chemistry 1999, 18, 664-672. 